\title{
SOLUTIONS FOR RADAR PULSE DEINTERLEAVING
}

by

Denis Kutman, B.Eng

SUBMITTED IN PARTIAL FULFILLMENT OF THE

REQUIREMENTS FOR THE DEGREE OF

MASTER OF APPLIED SCIENCE

AT

CARLETON UNIVERSITY

OTTAWA, ONTARIO

JANUARY 2011

(C) Copyright by Denis Kutman, 2011 


$\begin{array}{ll}\begin{array}{l}\text { Library and Archives } \\ \text { Canada }\end{array} & \begin{array}{l}\text { Bibliothèque et } \\ \text { Archives Canada }\end{array} \\ \begin{array}{l}\text { Published Heritage } \\ \text { Branch }\end{array} & \begin{array}{l}\text { Direction du } \\ \text { Patrimoine de l'édition }\end{array} \\ \begin{array}{l}\text { 395 Wellington Street } \\ \text { Ottawa ON K1A ON4 } \\ \text { Canada }\end{array} & \begin{array}{l}\text { 395, rue Wellington } \\ \text { Ottawa ON K1A ON4 } \\ \text { Canada }\end{array}\end{array}$

Your file Votre référence

ISBN: 978-0-494-81663-9

Our file Notre référence

ISBN: 978-0-494-81663-9

NOTICE:

AVIS:

The author has granted a nonexclusive license allowing Library and Archives Canada to reproduce, publish, archive, preserve, conserve, communicate to the public by telecommunication or on the Internet, loan, distribute and sell theses worldwide, for commercial or noncommercial purposes, in microform, paper, electronic and/or any other formats.

The author retains copyright ownership and moral rights in this thesis. Neither the thesis nor substantial extracts from it may be printed or otherwise reproduced without the author's permission.

L'auteur a accordé une licence non exclusive permettant à la Bibliothèque et Archives Canada de reproduire, publier, archiver, sauvegarder, conserver, transmettre au public par télécommunication ou par l'Internet, prêter, distribuer et vendre des thèses partout dans le monde, à des fins commerciales ou autres, sur support microforme, papier, électronique et/ou autres formats.

L'auteur conserve la propriété du droit d'auteur et des droits moraux qui protège cette thèse. $\mathrm{Ni}$ la thèse ni des extraits substantiels de celle-ci ne doivent être imprimés ou autrement reproduits sans son autorisation.
In compliance with the Canadian Privacy Act some supporting forms may have been removed from this thesis.

While these forms may be included in the document page count, their removal does not represent any loss of content from the thesis.
Conformément à la loi canadienne sur la protection de la vie privée, quelques formulaires secondaires ont été enlevés de cette thèse.

Bien que ces formulaires aient inclus dans la pagination, il n'y aura aucun contenu manquant.

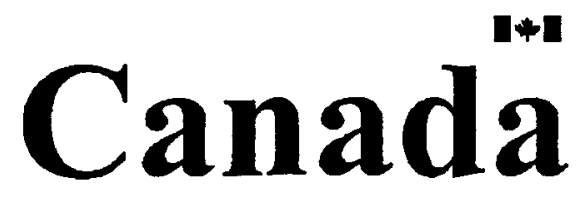


The undersigned recommend to the Faculty of Graduate Studies and Research acceptance of the thesis

\section{"Solutions for Radar Pulse Deinterleaving"}

submitted by Denis Kutman, B.Eng

in partial fulfillment of the requirements for the degree of Master of Applied Science.

Dr. Jim Wight, Thesis Supervisor

Dr. Huai-Jing Du, Thesis Co-supervisor

$\overline{\text { Dr. Qi-Jun Zhang, Chairman, Department of Electronics }}$

Carleton University

January 2011 


\section{Abstract}

The fields of Electronic Warfare (EW) and Signal Intelligence (SIGINT) have differing objectives but in many ways they complement each other. SIGINT systems primarily use a passive receiver for the gathering of long-term Electronic Intelligence (ELINT) and Communications Intelligence (COMINT). Algorithms that are used within the ELINT field must be capable of efficiently handling large amounts of data at one time. Since there is no requirement for real-time information, the data analysis may be done offline. This thesis proposes an offline solution that is capable of deinterleaving a large number of radar pulses. The offline solution consists of a three stage procedure. First, the pulses are sorted by their interpulse parameters, and then the next two stages use the intrapulse parameters to obtain a more refined grouping result. Conversely to ELINT, EW systems have active and passive components, and they can be used in an offensive or defensive manner. These systems must be able to process information in real-time so that decisions can be made instantaneously. This thesis will outline an online solution that is able to sort pulses in real-time as they are received. The online solution evaluates the similarity between pulses using their intrapulse characteristics. Both of the solutions are tested according to a simulated scenario, and they are shown to be successful at deinterleaving the pulses in all the test cases. 


\section{Acknowledgements}

First and foremost, I would like to thank my supervisors, Dr. Jim Wight and Dr. Huai-Jing Du, for their guidance and support throughout the preparation of this thesis. It was an honour and a privilege to have the chance to work with both of you.

I would also like to show my gratitude to Dr. Yifeng Zhou, Dr. Sreeraman Rajan and Dr. Fred Dilkes for the invaluable conversations and technical assistance. The information that you provided was crucial to the successful completion of this thesis.

Finally, I would like to thank my family and my girlfriend for their love and encouragement throughout my years of study. You helped me to maintain the focus and motivation to persevere through the difficult times. 


\section{Table of Contents}

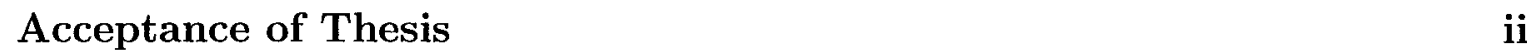

Abstract

Acknowledgements iv

Table of Contents $\quad$ v

List of Tables $\quad$ vii

List of Figures

$\begin{array}{ll}\text { List of Abbreviations } & \text { ix }\end{array}$

1 Introduction 1

1.1 Motivation . . . . . . . . . . . . . . . . . 4

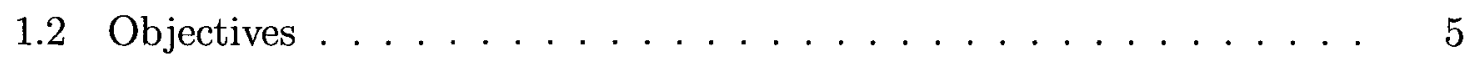

1.3 Organization of Thesis $\ldots \ldots \ldots \ldots \ldots$

2 Theory and Literature Review $\quad 7$

2.1 Radar Theory . . . . . . . . . . . . . . . . . . . . 7

2.2 Types of Radars . . . . . . . . . . . . . . . . . . . 9

2.3 General Pulse Signal Model . . . . . . . . . . . . . . . . . . . . 11

2.4 Radar Pulse Processing . . . . . . . . . . . . . . . . . . . . . . 13

2.5 Pulse Deinterleaving and Clustering . . . . . . . . . . . . . . 15

2.6 Scenario Definition . . . . . . . . . . . . . . . . 17

2.7 Literature Review . . . . . . . . . . . . . . . . . . 18

3 Algorithms 21

3.1 Emitter Number Estimation Algorithm . . . . . . . . . . . . 22

3.2 Pulse Comparator Algorithm _ . . . . . . . . . . . . . 30 
4 Proposed Solutions $\quad 36$

$\begin{array}{lll}5 & \text { Results } & 47\end{array}$

$\begin{array}{llr}6 & \text { Conclusion } & 67\end{array}$

$\begin{array}{ll}\text { Bibliography } & \mathbf{7 0}\end{array}$

$\begin{array}{ll}\text { Appendices } & 74\end{array}$

$\begin{array}{ll}\text { A The Radar Equation } & 75\end{array}$

$\begin{array}{ll}\text { B Signal-to-Noise Variations in LFM Pulse } & 78\end{array}$ 


\section{List of Tables}

5.1 Interpulse Parameters for Radar Systems in the Simulated Scenario . 49

5.2 Modulated Waveforms Used For Testing . . . . . . . . . . . . 57

5.3 Test Case Combinations . . . . . . . . . . . . . . . . . . . 58

5.4 Emitter Number Estimation Test Case A . . . . . . . . . . . . . 59

5.5 Emitter Number Estimation Test Case B . . . . . . . . . . . . 60

5.6 Emitter Number Estimation Test Case C . . . . . . . . . . . . . . . . 61

5.7 Pulse Comparator Test Case A _ . . . . . . . . . . . . . 63

5.8 Pulse Comparator Test Case B . . . . . . . . . . . . . . 64

5.9 Pulse Comparator Test Case C . . . . . . . . . . . . . . 65 


\section{List of Figures}

1.1 Overview of Electronic Warfare and Signal Intelligence $\ldots \ldots \ldots$

2.1 Radar Transmission Diagram . . . . . . . . . . . . . . . 8

2.2 Physical Scenario . . . . . . . . . . . . . . . . . . . . . 11

2.3 Deinterleaving Diagram . . . . . . . . . . . . . . . . . . . . 14

2.4 Electronic Warfare Signal Processing Steps . . . . . . . . . . . . . 15

2.5 K-Means Algorithm . . . . . . . . . . . . . . . . . . 16

4.1 Offline Solution . . . . . . . . . . . . . . . . . . . . . 37

4.2 Stage 1 Of The Offline Solution $\ldots \ldots \ldots$. . . . . . . . 39

4.3 Stage 2 Of The Offline Solution . . . . . . . . . . . . . 41

4.4 Stage 3 Of The Offline Solution . . . . . . . . . . . . . 43

4.5 Online Solution . . . . . . . . . . . . . . . . . . . . 45

5.1 Simulated Scenario . . . . . . . . . . . . . . . . . . . 48

5.2 Deinterleaving Using The Interpulse Parameters . . . . . . . . . . 51

5.3 No Modulation on the Pulse $(\mathrm{SNR}=21 \mathrm{~dB}) \ldots \ldots \ldots 53$

5.4 Linear Frequency Modulation $(\mathrm{LFM})(\mathrm{SNR}=21 \mathrm{~dB}) \ldots \ldots$

5.5 Frequency-Shift Keying $(\mathrm{FSK})(\mathrm{SNR}=21 \mathrm{~dB}) \ldots \ldots \ldots \ldots$

5.6 Phase-Shift Keying $(\mathrm{PSK})(\mathrm{SNR}=21 \mathrm{~dB}) \ldots \ldots \ldots$

B.1 Linear Frequency Modulation $(\mathrm{LFM})(\mathrm{SNR}=13 \mathrm{~dB}) \ldots \ldots \ldots$

B.2 Linear Frequency Modulation $(\mathrm{LFM})(\mathrm{SNR}=7 \mathrm{~dB}) \ldots \ldots$

B.3 Linear Frequency Modulation $(\mathrm{LFM})(\mathrm{SNR}=1 \mathrm{~dB}) \ldots \ldots . \ldots 81$ 


\section{List of Abbreviations}

$\begin{array}{ll}\text { AIC } & \text { Akaike Information Criteria } \\ \text { AR } & \text { Acquisition Radar } \\ \text { CLNN } & \text { Competitive Learning Neural Network } \\ \text { COMINT } & \text { Communications Intelligence } \\ \text { DOA } & \text { Direction Of Arrival } \\ \text { EA } & \text { Electronic Attack } \\ \text { ELINT } & \text { Electronics Intelligence } \\ \text { EM } & \text { Electromagnetic } \\ \text { EP } & \text { Electronic Protection } \\ \text { ES } & \text { Electronic Support } \\ \text { EW } & \text { Electronic Warfare } \\ \text { EWR } & \text { Early Warning Radar } \\ \text { FSK } & \text { Frequency-Shift Keying } \\ \text { IFF } & \text { Identification Friend or Foe } \\ \text { IID } & \text { Independent and Identically Distributed } \\ \text { IMOP } & \text { Intentional Modulation On Pulse } \\ \text { LFM } & \text { Linear Frequency Modulation } \\ \text { LPI } & \text { Low Probability of Intercept } \\ \text { MATLAB } & \text { Matrix Laboratory } \\ \text { MDL } & \text { Minimum Description Length } \\ \text { MTI } & \text { Moving Target Indicator } \\ \text { NOMOD } & \text { No Modulation } \\ \text { PDW } & \text { Pulse Descriptor Words }\end{array}$




$\begin{array}{ll}\text { PRI } & \text { Pulse Repetition Interval } \\ \text { PSK } & \text { Phase-Shift Keying } \\ \text { PW } & \text { Pulse Width } \\ \text { RADAR } & \text { Radio Detection and Ranging } \\ \text { RF } & \text { Radio Frequency } \\ \text { RWR } & \text { Radar Warning Receiver } \\ \text { SAR } & \text { Synthetic Aperture Radar } \\ \text { SEI } & \text { Specific Emitter Identification } \\ \text { SIGINT } & \text { Signal Intelligence } \\ \text { SNR } & \text { Signal-To-Noise Ratio } \\ \text { SOMNN } & \text { Self-Organizing Feature Map Neural Network } \\ \text { SVC } & \text { Support Vector Clustering } \\ \text { TOA } & \text { Time Of Arrival } \\ \text { TR } & \text { Tracking Radar } \\ \text { UMOP } & \text { Unintentional Modulation On Pulse }\end{array}$




\section{Chapter 1}

\section{Introduction}

Electronic Warfare (EW) refers to any action taken to gain control of the Electromagnetic (EM) spectrum. The purpose of EW is to allow the user to perform active and passive EM sensing while denying the same functionality to the enemy [1]. Electronic Warfare has been a very popular field of research since the invention of Radio Detection and Ranging (Radar) in the 1930's, prior to World War II. Radar systems have been continuously evolving, increasing in complexity throughout the decades. In order to maintain its effectiveness, EW equipment must follow a similar path of progression.

The area of Electronic Warfare can be split into three sub-disciplines called Electronic Support (ES), Electronic Attack (EA) and Electronic Protection (EP) [1]. EA and EP systems include any sort of electronic devices designed to trick, deceive or counter radar, sonar, or other detection platforms. The goal of deception can be achieved by either the use of jamming or decoys. EA and EP are active systems and may be used to deny targeting information to an enemy in an 


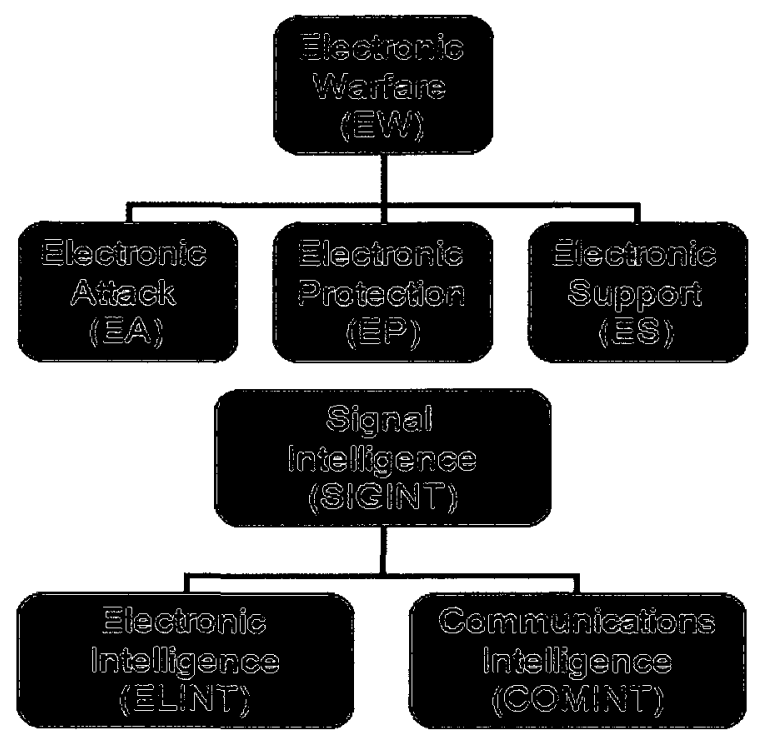

Figure 1.1: Overview of Electronic Warfare and Signal Intelligence

offensive or defensive manner. Conversely, ES systems are typically passive, and they are mainly used for gathering data which is subsequently used by a platform for situational awareness [1]. For example, Radar Warning Receivers (RWRs) installed on fighter jets are designed to give immediate warning to the pilot if there is a threat in the vicinity. The warning receiver performs all the processing and decision-making in real-time.

The Signal Intelligence (SIGINT) field is typically used in conjunction with EW equipment to obtain a more accurate picture of the type of emitters and where they are located in the surrounding environment. ES and SIGINT systems have a lot of similarity but differ in the way that their output data is utilized. The output of ES systems is used to take immediate action, while the output from a SIGINT receiver is gathered over a lengthy period of time and analyzed offline. SIGINT can be divided into Communications Intelligence (COMINT) and Electronic Intelligence 
(ELINT). COMINT is used to examine the message that is contained within a signal while ELINT is not interested in the message that is contained within the incoming signal [1]. With respect to the SIGINT field, this thesis will focus primarily on the part that is related to ELINT.

ELINT refers to the information that is gained from signals of interest as they are intercepted [2]. ELINT data can be extremely valuable in the event of a conflict. Once the signal is identified, the ELINT receiver will then analyze it and decide whether the source could be a potential threat [3]. Knowledge regarding the type of source emitter and its location can provide a clearer picture of the situation in a hostile environment. The data obtained from the ELINT receiver could enable intelligent jamming of an enemy's defense network. ELINT can also be utilized in stealth operations, providing the stealth aircraft with information on which areas to avoid. In addition to radar, it is possible to gather ELINT data from other sources such as beacons, transponders, jammers, missile guidance, altimeters, navigation emissions, and Identification Friend or Foe (IFF) [2]. Although ELINT from other types of signals may be valuable, this thesis will focus on gathering information from signals originating from radar systems. Unless otherwise specified, any references to EW in this thesis will include the field of ELINT because of the similarity between the two subjects. 


\subsection{Motivation}

Radar system designs are continuously being modified to reflect the newest technological innovations of the day. Present systems are more compact and offer a wider variety of capabilities when compared with older designs. Some of the new features available in the current systems include frequency agility, pulse compression as well as other complex modulation techniques. New developments such as the Low Probability of Intercept (LPI) radars are providing significant challenges for some of the existing EW receivers. LPI radar has a number of features which make it very hard to identify including low sidelobe levels, infrequent scanning, very high duty cycles and frequency hopping. This increasingly complex emitter environment makes it difficult for the current radar pulse sorting techniques to maintain their effectiveness. Since the emitter threat constantly varies, the EW receiver must be able to adapt to this continuously changing environment [2].

The conventional methods for sorting used the interpulse parameters to allocate pulses to certain emitters, but these methods have a number of limitations. The Radio Frequency (RF) feature would not be efficient at identifying pulses that come from emitters with frequency agility. The Direction of Arrival (DOA) feature may classify pulses incorrectly if there are reflected signals arriving at the receiver. The Pulse Width (PW) may not be reliable because of multipath effects and thresholding problems relating to varying pulse amplitudes. The Pulse Repetition Interval (PRI) feature will have trouble identifying emitters that have PRI staggering. Apart from all these potential issues, another large problem occurs when platforms in the vicinity have the same radar systems with the same interpulse 
characteristics. In this scenario, the pulse sorting mechanism would be unable to differentiate between the emitters [2].

In battlefield situations, it is becoming more common to see sides using identical platforms or unusual, exotic emitters. The classical signal identification techniques which used interpulse parameters for interception and deinterleaving need to be extended to handle the increasing complexity of some of the current and future radar waveforms. This extension may be completed by using the information extracted from the intrapulse characteristics of the signal [4]. The EW receiver must be capable of exploring characteristics inside each pulse, such as the shape of the envelope as well as the frequency and phase variations. All individual emitters have their own distinct electrical signal structure inside each of their transmitted pulses. This can be due to both intentional and unintentional modulations. Since no transmitting device is perfect, there is usually inherent ringing or other instabilities that lead to unintentional modulations [5].

\subsection{Objectives}

The goal of this thesis is to outline possible solutions that can be used, within the fields of Electronic Warfare and Signal Intelligence, to deinterleave a set of received pulses. Prior to the discussion of any solutions, the general problem will be explained, and the formal scenarios will be introduced. Then, the steps for each solution will be described and validated through testing and simulation to show how they can be used to solve the problem. 


\subsection{Organization of Thesis}

The rest of this thesis is organized as follows. In Chapter 2, background information will be provided so that the topics discussed in this thesis are easier to comprehend for the reader. Chapter 3 contains a detailed derivation of the two algorithms that are essential to the proposed solutions. Chapter 4 outlines the overall offline and online solutions in detail. In Chapter 5, the solutions are tested to show that they are effective at performing the tasks that they are assigned. Then, Chapter 6 will summarize the findings in this thesis and outline possible topics for future research that may be carried on following this thesis. 


\section{Chapter 2}

\section{Theory and Literature Review}

\subsection{Radar Theory}

A radar functions by radiating Electromagnetic (EM) energy and detecting the echo that is returned from reflected objects or targets. Using the fact that radar signals travel at the speed of light, the distance of a target from the radar is found by measuring the time it takes for the radiated energy to travel to the target and back. The angular location or azimuth of the target can also be found using the directive radar antenna, which typically has a narrow beamwidth. The radar system is able to track moving targets by taking advantage of the Doppler Effect. The received echo signal from a moving target such as an aircraft will have a shift in the frequency. The radar uses this shift in frequency to differentiate between the aircraft and the other stationary clutter [6]. 
Radar system designs vary depending on the objective of the user. The radar manufacturer has control over parameters such as the power, gain, pulse width, frequency, pulse repetition interval and the pulse waveform. A derivation of the radar equation is provided in Appendix A that outlines some of the important parameters to consider when designing a radar. Appendix A also shows how the radar equation can be applied to the EW scenario. In this case, the one-way radar equation is used for analysis [6].

Figure 2.1 outlines the radar transmission cycle. The cycle starts with the radar transmitting a pulse. It then listens for echoes for a set amount of time until it is ready to transmit the next pulse. The dead time is the amount of time it takes the radar to transmit the pulse, and the pulse repetition interval is the amount of time between pulse transmissions [6].

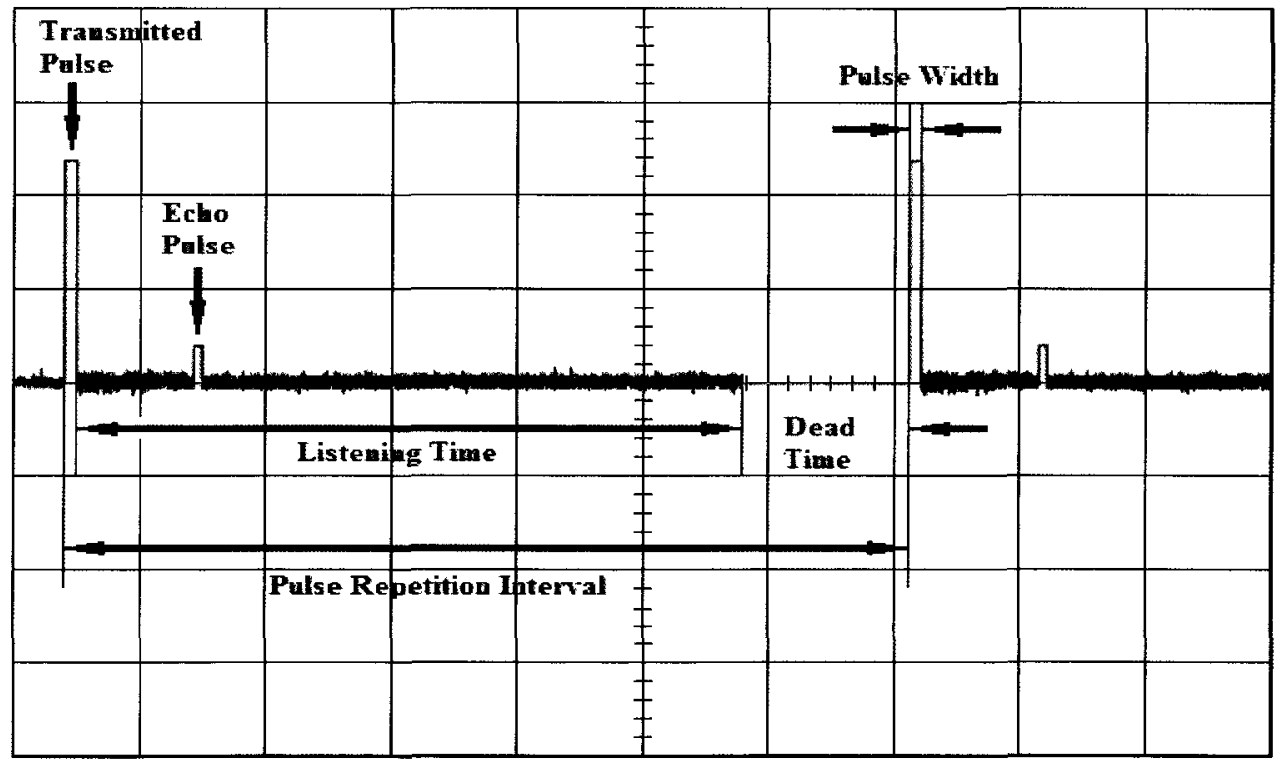

Figure 2.1: Radar Transmission Diagram 


\subsection{Types of Radars}

There are many different types of radar systems in use today. Each has its own purpose, and every system has advantages and disadvantages. Military organizations use radar technology in many applications including surveillance, navigation, tracking, terrain mapping, fire control and weather observation. Three categories of radar systems will be discussed that are relevant to the simulated scenario that is presented later in this thesis.

\section{Search Radars}

Air search radars are sometimes called early warning radars because they are used to provide an early notification to the user that a target is approaching. Search radars are ground-based systems, and they are used to monitor all of the 360 degree azimuth sectors within the airspace. These radars typically have maximum ranges of between $200 \mathrm{~km}$ and $500 \mathrm{~km}$. They have relatively low frequencies, long pulse durations, and they transmit with a large amount of power. In order to improve the range resolution, most search radars use a technique called pulse compression, which uses a chirped (linear frequency modulated) signal. This type of radar is not considered to be a direct threat because it is only used for the location of targets. Once the search radar acquires a target, it usually passes the information on to a weapons system for further investigation. Its high power emissions make it easy to locate and characterize using an EW receiver [7]. 


\section{Battlefield Surveillance Radars}

There are many types of radars that may potentially be used in a battlefield to perform the surveillance function. Some of these include Acquisition radars, Doppler radars, Synthetic Aperture Radars (SAR), and Low Probability of Intercept (LPI) radars. Acquisition radars have many similarities with the air search radars except that they typically have smaller ranges to cover. A common maximum range for an acquisition radar is around $100 \mathrm{~km}$ to $150 \mathrm{~km}$. Doppler radars can detect and locate vehicles and personnel moving over the ground using a Moving Target Indicator (MTI) system. Synthetic Aperture Radars can be used to map high resolution terrain using data that is combined over a period of time. LPI Radars are designed in a way which makes them very hard to detect by an enemy EW suite [7].

\section{Tracking Radars}

Tracking radars provide frequent target location updates and high accuracy position estimates to allow the weapons system to engage the target. In order to achieve this high positioning accuracy and fast update rate, they have short pulse widths, small pulse repetition intervals, and they use narrow pencil beams to scan a specific part of the airspace. In many situations, an air search radar or acquisition radar is used to initially detect the target and forwards the information to the tracking radar to monitor the target more closely [7]. 


\subsection{General Pulse Signal Model}

Similarly to [5] and [8], a general pulse signal model outlining a possible emitter deinterleaving scenario is illustrated in Figure 2.2 below. The diagram contains $\mathrm{K}$ independent emitters surrounding the passive EW receiver. This intercept receiver collects the pulses as they arrive, interleaved in time.

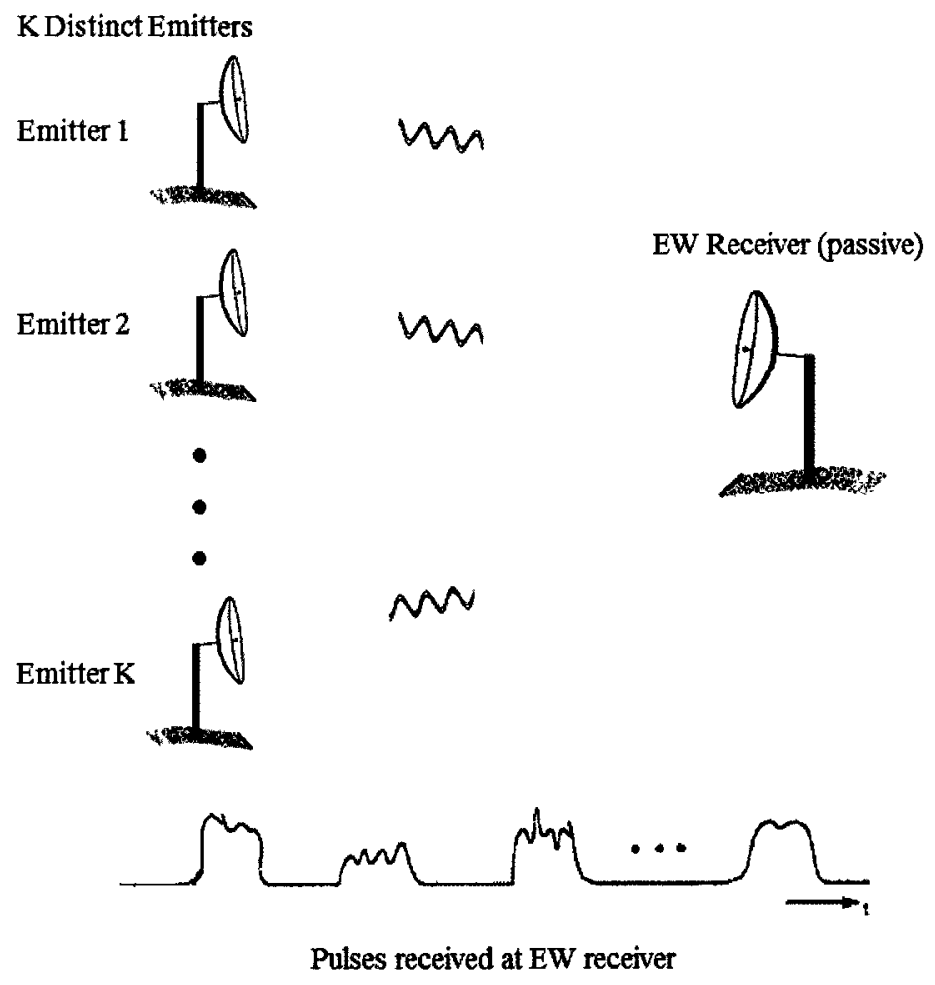

Figure 2.2: Physical Scenario

To formulate the general pulse signal model, it is assumed that the receiver receives a total of $\mathrm{M}$ pulses. The $m^{\text {th }}$ received pulse can then be designated by $x_{m}\left(t ; q_{m}\right)$, where $\mathrm{m}=1,2, . ., \mathrm{M}$ and $q_{m}$ is the association parameter that associates the $m^{\text {th }}$ pulse with the $k^{\text {th }}$ emitter (i.e. $q_{m}=\mathrm{k}$ ). 
The $m^{\text {th }}$ received pulse can be expressed as follows:

$$
x_{m}\left(t ; q_{m}\right)=A_{m} a_{q_{m}}\left(t-\tau_{m}\right) e^{\jmath\left[\psi_{m}+\omega_{m}\left(t-\tau_{m}\right)+\phi_{q_{m}}\left(t-\tau_{m}\right)\right]}+v_{m}\left(t-\tau_{m}\right)
$$

where:

- $A_{m}=$ initial amplitude of the $m^{\text {th }}$ received pulse

- $a_{q_{m}}=$ original envelope of the $m^{t h}$ received pulse

- $\tau_{m}=$ time delay of the $m^{\text {th }}$ received pulse with respect to the reference

- $\psi_{m}=$ initial phase of the $m^{t h}$ received pulse

- $\omega_{m}=$ carrier frequency of the $m^{\text {th }}$ received pulse

- $\phi_{q_{m}}=$ original phase of the $m^{\text {th }}$ received pulse

- $v_{m}=$ gaussian noise with the $m^{\text {th }}$ received pulse

The equation above has a number of nuisance parameters including $A_{m}, \tau_{m}$, $\psi_{m}$, and $\omega_{m}$. Some reports related to radar emitter classification [5] [8] [9] [10] [11] require that all of these nuisance parameters be removed through pre-processing prior to performing the classification. In [10], the amplitude variations are removed using normalization techniques, while the phase variations are removed using polynomial fitting. The solutions proposed in this thesis require time alignment and carrier frequency removal, but they do not require any pre-processing in the phase or amplitude prior to the deinterleaving. In order to compare two pulses, they must first be aligned in time (i.e. the time delay, $\tau_{m}$, must be removed). The time delay is caused by the triggering mechanism of the receiver. Since incoming signals are 
normally corrupted by noise and may have significantly varying signal-to-noise ratios, there may be different time delays associated with the pulses. Some common methods of removing this time delay include aligning pulses to the point at a power level $6 \mathrm{~dB}$ below the peak [12], using a pre-set magnitude threshold value [10] and performing a cross-correlation between the pulses [13]. The input noise term, $v_{m}$, includes the medium ambient noise, antenna thermal noise and the circuitry noise but is typically dominated by the circuitry noise.

\subsection{Radar Pulse Processing}

Radar pulse processing within the EW field consists of a number of intermediate steps. The first step involves the interception and detection of pulse trains from radar systems in the surrounding environment. Following the detection stage, the pulse parameters must be estimated. This estimation is completed by extracting the interpulse and intrapulse parameters from each individual pulse and storing them in a vector format. The interpulse parameters can also be referred to as Pulse Descriptor Words (PDWs). The classical PDWs typically include the Radio Frequency (RF), Pulse Width (PW), Time Of Arrival (TOA), Direction Of Arrival (DOA) and Pulse Repetition Interval (PRI). The intrapulse information can be extracted as a set of complex samples that represent the pulse. Since the various signals are interleaved when they arrive at the EW receiver, there may be pulses from many different emitters, and successive pulses may not have originated from the same source. Therefore, after the pulse parameters are estimated, the receiver completes a process called deinterleaving which attempts to separate the pulses into 
groups from the same emitter [14]. An example of deinterleaving is shown below in Figure 2.3.

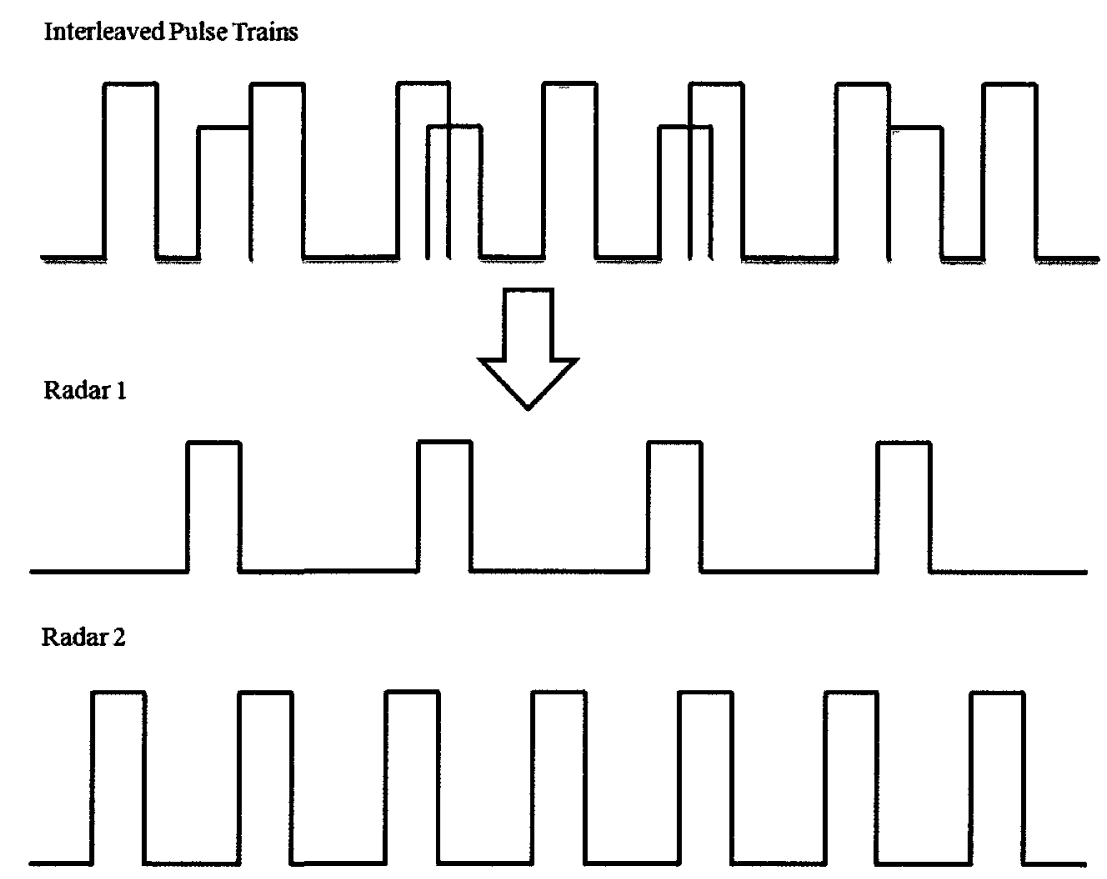

Figure 2.3: Deinterleaving Diagram

Following this sorting, there is an attempt to identify or correlate the pulse with a database of known emitters. This could provide an indication of whether the pulse came from a platform that could be a threat. The final step requires some action to be performed. The action may be passive such as displaying or recording the result, or it may be a direct countermeasure procedure such as jamming or chaff. ES and ELINT systems rarely initiate a countermeasure action since they are used more for gathering data passively, while EA and EP systems are capable of initiating an action in an offensive or defensive manner [14]. Figure 2.4 below illustrates the steps involved in the radar pulse processing. 


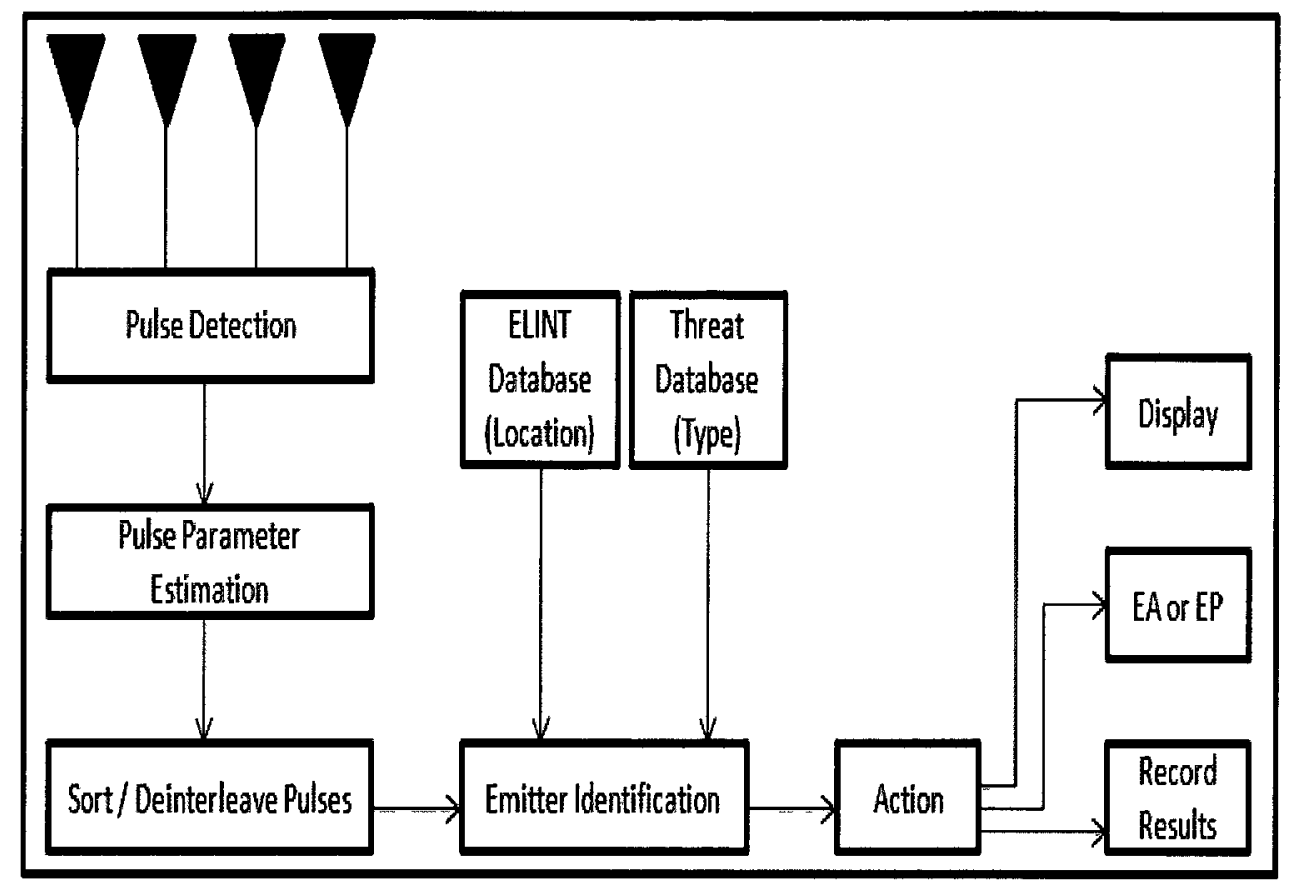

Figure 2.4: Electronic Warfare Signal Processing Steps

\subsection{Pulse Deinterleaving and Clustering}

Similar to the receiver block of a radar system, the EW receiver is largely statistics-based. Once the vectors are obtained from the pulse deinterleaver, they are used to create a statistical model of the emitters in the surrounding area. This model is created using a technique called cluster analysis or clustering. Cluster analysis is an unsupervised learning technique which attempts to find structure or commonality within a collection of unlabeled data. Clustering involves the process of organizing objects into groups whose members are similar in some way. There are a wide variety of methods that can be used for clustering a data set. Each algorithm has its own advantages and disadvantages depending on the application where it is used [15]. 
There are many clustering techniques that may be used to sort an unlabeled data set. Some of the more common methods include K-Means and Hierarchical Clustering. These algorithms are used in many real-world applications because of their simplicity and effectiveness. Part of the offline solution defined in this thesis uses K-Means Clustering to initially partition the data set. The K-Means algorithm is outlined below in Figure 2.5. The iterative procedure is used to find the optimal means by minimizing a squared-error criterion function. The resulting optimal means that are obtained may be accepted as the final answer, or they can be used as starting points for more exact computations [15].

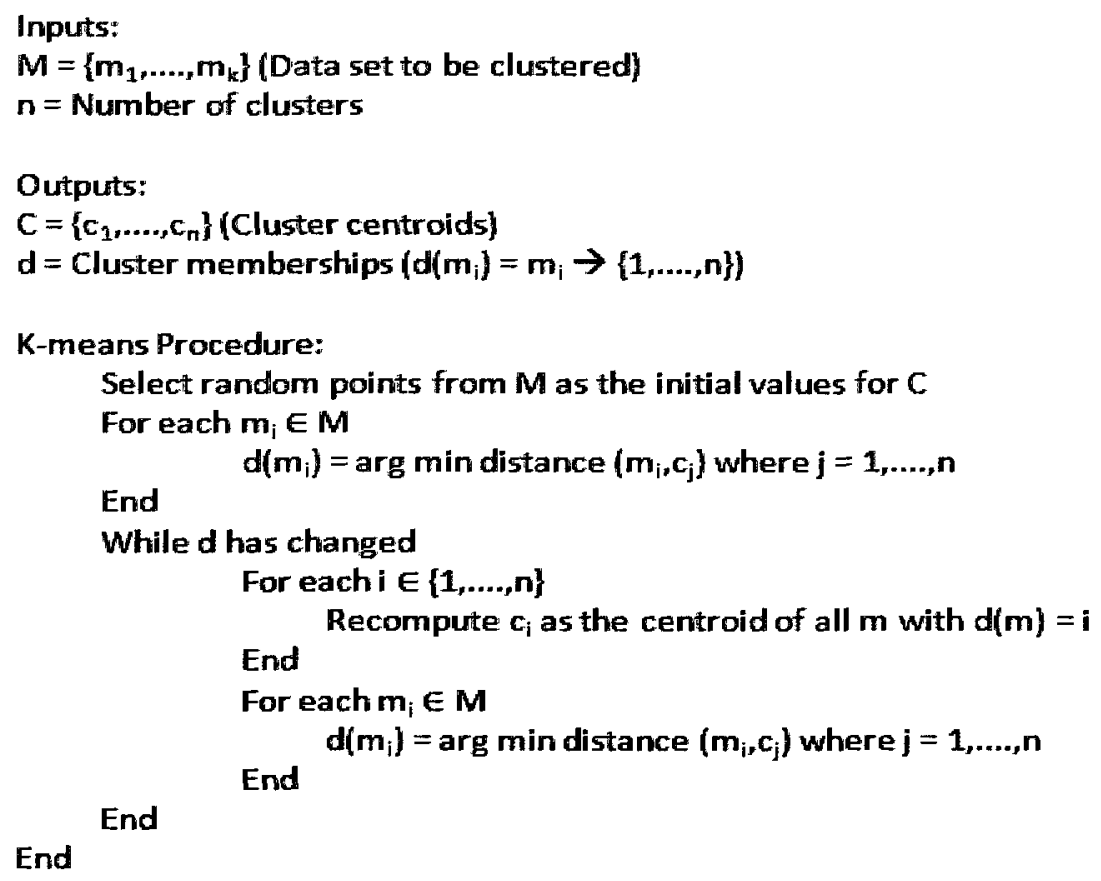

Figure 2.5: K-Means Algorithm 


\subsection{Scenario Definition}

There are a number of different scenarios that could occur in the field when an EW receiver is deployed and operational. This thesis formally proposes two realistic scenarios that could potentially arise. These scenarios are listed below:

\section{Scenario 1: Emitters have varying interpulse parameters}

This is the classical EW problem where emitter identification and classification needs to be completed. In this case, using the various interpulse parameters such as the Radio Frequency (RF), Pulse Width (PW), Time Of Arrival (TOA), Direction Of Arrival (DOA) and Pulse Repetition Interval (PRI) should be sufficient to complete this detection and sorting.

Scenario 2: Emitters have common interpulse parameters and varying intrapulse parameters

This scenario is becoming more common in the present EW environment. It is possible to have multiple emitters from the same manufacturer or emitters with very unusual pulse characteristics. It's clear that the conventional solution that could be utilized for scenario 1 would not be adequate in this situation. 


\subsection{Literature Review}

There are a number of different solutions that have been proposed for the two scenarios described above. For the first scenario, each pulse can be described with a vector of PDW attributes. Clustering algorithms would then use these vectors to sort the pulses into individual clusters. In [16], clustering is performed using a combination of Support Vector Clustering (SVC) and the K-Means algorithm. After the PDW parameters are extracted from each radar pulse, the SVC method uses a small sample of the PDW feature vectors to obtain the initial centroids that are then used by the K-Means algorithm to classify the full data set. In [17], the pulses are first sorted using PDWs such as the RF, DOA, and PW. Then, the second stage of sorting is done by using a difference histogram to extract the PRI from the TOA measurements.

For the second scenario that was described in the previous section, each pulse is typically described as a vector of complex samples. In this case, the pulse deinterleaving is completed using the intrapulse data. In [18], three unsupervised classifiers including Competitive Learning Neural Networks (CLNN), Self-Organizing Feature Map Neural Networks (SOMNN) and Support Vector Clustering (SVC) are used to sort radar pulses based on their intrapulse characteristics. In [19], four different self-organizing neural networks were used for automatic clustering of radar pulses. In [9], [10], [8], the pulse classification task was formulated as a multivariate clustering problem, and the Minimum Description Length (MDL) criteria was used to perform the cluster validation. 
Techniques have also been proposed in [11] and [13] which are able to estimate the modulations for a group of received radar pulses. In [11], a computationally efficient algorithm is proposed that makes use of a maximum likelihood estimator. In [13], the M-estimation technique is proposed, and it is shown to be especially effective in situations where there are difficulties encountered in the pulse pre-processing stage.

Specific Emitter Identification (SEI) is one of the newer concepts that has been introduced within the field of Electronic Warfare. SEI refers to having the capability to associate a received pulse waveform with a unique emitter. Each emitter has its own individual electrical signature inside each of its transmitted pulses due to both intentional and unintentional modulations. The goal of SEI is similar to fingerprinting, in that the characteristics of every individual radar emitter are recorded and stored in a library for future reference [4]. As new pulses are received and processed, their signatures are compared to others in the current library to see if there is a match. In cases where there is no match, a new entry is added to the library with the appropriate pulse information. In [5], intrapulse features are used to implement the SEI process. After the intrapulse features are extracted from each pulse, the feature selection is performed. Feature selection is the process of choosing the subset of the original features that is the most predictive, eliminating redundant and uninformative ones. In [4], the SEI process uses the Unintentional Modulation on Pulse (UMOP) features to augment the classical interpulse parameter classification. 


\section{Chapter Summary}

This chapter was included to provide the reader with background information that relates to the main topics covered in this thesis. First, some of the basic theory for radar system operation was discussed. Then, the physical scenario for an EW receiver was outlined with the aid of the general pulse signal model representation. The next two sections described the various stages that are involved in the processing of radar pulses. Finally, two formal scenarios were defined followed by a discussion of some of the existing solutions for each scenario. The next chapter will describe the algorithms that will be part of the proposed solution in this thesis. 


\section{Chapter 3}

\section{Algorithms}

In this chapter, two algorithms are outlined that could be combined to create a possible solution for the second scenario that was described in the previous chapter. The first algorithm that will be described is called the Emitter Number Estimation algorithm [20]. Given a set of received pulse trains, this algorithm can be used to estimate the number of emitters that the pulse trains originated from. The second algorithm to be described is called the Pulse Comparator algorithm [21]. This algorithm is used to compare the likelihood that two pulses come from the same emitter. 


\subsection{Emitter Number Estimation Algorithm}

The Emitter Number Estimation algorithm that is introduced in this thesis uses the Information Theoretic Approach. The Information Theoretic Criteria have been used in the field of data analysis for many years. They are typically used to select the optimum model to represent a data set. The Information Theoretic Criteria is based on the application of the algorithms that were introduced by Akaike [22], Rissanen [23] and Schwartz [24]. In model selection problems, the Information Theoretic Criteria take into account both the goodness-of-fit (likelihood) of a model and the number of parameters used to achieve that fit. The criteria take the form of a penalized likelihood function consisting of a negative log-likelihood term added to a penalty function [25]. There have been a number of papers written on the topic of signal/emitter number estimation using the Information Theoretic Criteria including [12], [20], [25], [26], [27] and [28].

In [20], an algorithm based on the Information Theoretic Criteria is proposed for the detection of the number of signals that is received by an array of sensors. This approach uses the assumption that the number of signals may be determined using the eigenvalues of the covariance matrix of the observation vector. The major advantage of this approach is that there is no subjective threshold selection required in the decision-making process. The number of signals is obtained by finding the value that minimizes the Akaike Information Criteria (AIC) or the Minimum Description Length (MDL) criteria. This thesis applies these criteria to the problem of radar emitter number estimation. This section will outline the pulse signal model and provide a derivation for the Emitter Number Estimation algorithm. 
First, the pulse signal model is formulated according to [28]. For the formulation, it is assumed that there are $\mathrm{K}$ emitters transmitting narrow band pulse signals. The following baseband representation for the $m^{\text {th }}$ pulse signal assumes that it comes from the $k^{\text {th }}$ emitter.

$$
x_{m}(t)=\delta_{m} s_{k}\left(t-\tau_{m}\right)+w_{m}\left(t-\tau_{m}\right)
$$

where:

- $s_{k}(t)=$ complex pulse waveform from $k^{t h}$ emitter

- $\delta_{m}=$ amplitude and phase shift on the received pulse

- $\tau_{m}=$ time delay of the $m^{\text {th }}$ pulse with respect to the reference

- $w_{m}(t)=$ gaussian noise with the $m^{t h}$ received pulse

As discussed in the previous chapter, the time delay is a nuisance parameter, and it can be removed by aligning the pulses with a common reference point. The alignment errors can be ignored if the sampling rate of the receiver is sufficiently high. After removing the time delay, the previous signal model becomes:

$$
x_{m}(t)=\delta_{m} s_{k}(t)+w_{m}(t)
$$

It is assumed that the signal waveforms, $s_{k}(t)$, are unknown deterministic and the noise process $w_{m}(t)$ is an additive and Independent and Identically Distributed (IID) Gaussian process with zero mean and an unknown variance of $\sigma_{w}^{2}$. Putting the previous equation into vector format, the $m^{\text {th }}$ pulse model is:

$$
x_{m}=\delta_{m} s_{k}+w_{m}
$$


Where $x_{m}=\left[x_{m}(1), \ldots . ., x_{m}(N)\right], s_{k}=\left[s_{k}(1), \ldots . ., s_{k}(N)\right], w_{m}=\left[w_{m}(1), \ldots . ., w_{m}(N)\right]$ and $\mathrm{N}$ is the number of samples representing a pulse.

Using the pulse signal formulation, the derivation for the Emitter Number Estimation algorithm will be completed using [20]. Writing the equation above in matrix form:

$$
X=A S+W
$$

Where $\mathrm{X}=\left[x_{1}, x_{2}, \ldots, x_{M}\right]^{T}, \mathrm{~S}=\left[s_{1}, s_{2}, \ldots, s_{K}\right]^{T}, \mathrm{~W}=\left[w_{1}, w_{2}, \ldots ., w_{M}\right]^{T}$ and $\mathrm{A}$ is the association matrix of size $M \times K$. Each row of A has exactly one non-zero entry associating the received pulse with an emitter. This means that the columns of A are linearly independent, and that A has a full column rank of $\mathrm{K}$.

The covariance matrix of $\mathrm{X}$ can be written as $\mathrm{R}=\mathrm{E}\left[(\mathrm{X})(X)^{\dagger}\right]$, with $\dagger$ referring to the conjugate transpose. The following formula is obtained:

$$
R=E\left[(A S+W)(A S+W)^{\dagger}\right]
$$

Since the noise is zero mean and independent of the pulse waveforms the equation above can be simplified to the following:

$$
R=\Psi+\sigma_{w}^{2} I
$$

Where:

$$
\Psi=A \tilde{S} A^{\dagger}
$$

Since the covariance matrix of the pulse waveforms, $\tilde{S}=S S^{\dagger}$, is assumed to be nonsingular, it can be observed that the rank of $\Psi$ is $\mathrm{K}$. This means that the $M-K$ smallest eigenvalues of $\Psi$ are equal to zero. The eigenvalues of $\mathrm{R}$ can be 
described in decreasing order as $\lambda_{1} \geq \lambda_{2} \geq \cdots \geq \lambda_{M}$, and the smallest $M-K$ eigenvalues of $\mathrm{R}$ are equal to $\sigma_{w}^{2}$ (ie: $\lambda_{K+1}=\lambda_{K+2}=\cdots \lambda_{M}=\sigma_{w}^{2}$ ). This means that the number of emitters can be estimated from the multiplicity of the smallest eigenvalue of $\mathrm{R}$. In practice, the actual covariance matrix $\mathrm{R}$ is typically unknown, and it must be estimated using a finite set of samples. As a result, the eigenvalues that are obtained will all be different, making it difficult to determine the number of emitters simply by observing the eigenvalues.

Given a set of observations and a family of models or a parametrized family of probability densities, the Information Theoretic Criteria attempt to select the model that best fits the data. Akaike [22] proposed that the model which gives the minimum AIC should be selected. The AIC is defined as:

$$
A I C=-2 \log f(X \mid \hat{\Theta})+2 p
$$

Where $\mathrm{X}$ is the set of observations, $\hat{\Theta}$ is the maximum likelihood estimate of the parameter vector $\Theta$ and $p$ is the number of free adjusted parameters within the model. The first term in the AIC equation is the log-likelihood of the maximum likelihood estimator and the second term is a bias correction term that makes the AIC an unbiased estimator.

Rissanen [23] and Schwartz [24] proposed that the model which gives the minimum MDL should be selected. The MDL criterion is given by:

$$
M D L=-\log f(X \mid \hat{\Theta})+\frac{1}{2} p \log N
$$

It can be observed that the first term of the MDL criterion is the same as the first term of the AIC except for the factor of two and the second term of the MDL criterion is only slightly different than the second term for the AIC. 
In order to apply the Information Theoretic Criteria to detect the number of emitters, the family of competing models must be defined. The observations will be regarded as Independent and Identically Distributed (IID) complex Gaussian random vectors with zero means. The family of models will be represented by the following family of covariance matrices:

$$
R_{\jmath}=\Psi_{\jmath}+\sigma^{2} I
$$

Where $\Psi_{j}$ is a matrix with an unknown rank $\mathrm{j}$ such that $j \in[0,1, \ldots, \mathrm{M}-1]$ and $\sigma$ is an unknown scalar quantity.

Using the spectral representation theorem from linear algebra, $R_{\jmath}$ can be expressed as:

$$
R_{\jmath}=\sum_{\imath=1}^{\jmath}\left(\lambda_{\imath}-\sigma^{2}\right) V_{\imath} V_{\imath}^{\dagger}+\sigma^{2} I
$$

Where $\lambda_{1}, \lambda_{2}, \ldots, \lambda_{\jmath}$ and $V_{1}, V_{2}, \ldots, V_{\jmath}$ are the eigenvalues and eigenvectors of $R_{\jmath}$, respectively. Therefore, the parameter vector $\Theta_{\jmath}^{T}=\left(\lambda_{1}, \lambda_{2}, \ldots, \lambda_{\jmath}, V_{1}^{T}, V_{2}^{T}, \ldots, V_{\jmath}^{T}, \sigma^{2}\right)$

Given the Multivariate Gaussian Density Function:

$$
f_{x}(x)=\frac{1}{(2 \pi)^{\frac{N}{2}} \sqrt{\operatorname{det} R_{\jmath}}} \exp \left[-\frac{1}{2}\left(x^{\dagger}\left(R_{\jmath}\right)^{-1} x\right)\right]
$$

The joint probability density for $\mathrm{N}$ observations which are IID complex Gaussian random vectors with zero mean is given by:

$$
f\left(x_{1}, x_{2}, \ldots, x_{M} \mid \Theta_{\jmath}\right)=\prod_{\imath=1}^{N} \frac{1}{(2 \pi)^{\frac{N}{2}} \sqrt{\operatorname{det} R_{\jmath}}} \exp \left[-\frac{1}{2}\left(x_{\imath}^{\dagger}\left(R_{\jmath}\right)^{-1} x_{\imath}\right)\right]
$$

The likelihood function is then given by:

$$
L\left(\Theta_{\jmath}\right)=[\text { constant }] \prod_{\imath=1}^{N} \operatorname{det}\left(R_{\jmath}\right)^{-\frac{1}{2}} \exp \left[-\frac{1}{2}\left(x_{\imath}^{\dagger}\left(R_{\jmath}\right)^{-1} x_{\imath}\right)\right]
$$


Simplifying and leaving out terms that do not depend on $\Theta_{\jmath}$ :

$$
\begin{aligned}
L\left(\Theta_{\jmath}\right) & =\operatorname{det}\left(R_{\jmath}\right)^{-\frac{N}{2}} \exp \left[-\frac{1}{2} \sum_{\imath=1}^{N} x_{\imath}^{\dagger}\left(R_{\jmath}\right)^{-1} x_{\imath}\right] \\
& =\operatorname{det}\left(R_{\jmath}\right)^{-\frac{N}{2}} \exp \left[-\frac{1}{2} \sum_{\imath=1}^{N} \operatorname{tr}\left(x_{\imath}^{\dagger}\left(R_{\jmath}\right)^{-1} x_{\imath}\right)\right] \\
& =\operatorname{det}\left(R_{\jmath}\right)^{-\frac{N}{2}} \exp \left[-\frac{1}{2} \operatorname{tr}\left(\left(R_{\jmath}\right)^{-1} \sum_{\imath=1}^{N} x_{\imath} x_{\imath}^{\dagger}\right)\right]
\end{aligned}
$$

Simplifying and taking the logarithm of the likelihood function to get the log-likelihood:

$$
L\left(\Theta_{\jmath}\right)=-N \log \left[\operatorname{det}\left(R_{\jmath}\right)\right]-\operatorname{tr}\left(\left(R_{\jmath}\right)^{-1} \hat{R}\right)
$$

Where $\hat{R}$ is the sample covariance matrix defined by:

$$
\hat{R}=\frac{1}{N} \sum_{\imath=1}^{N} x_{\imath} x_{\imath}^{\dagger}
$$

The maximum likelihood estimate is the value of $\Theta_{\jmath}$ that minimizes the formula above. Using the estimates obtained in [29]:

$$
\begin{aligned}
& \hat{\lambda}_{\imath}=l_{\imath} \quad \mathrm{i}=1, \cdots, \mathrm{j} \\
& \hat{\sigma^{2}}=\frac{1}{M-j} \sum_{\imath=\jmath+1}^{M} l_{\imath} \\
& \hat{V}_{\imath}=C_{\imath} \quad \mathrm{i}=1, \cdots, \mathrm{j}
\end{aligned}
$$

Where $l_{1}>l_{2}>\cdots>l_{M}$ and $C_{1}, C_{2}, \cdots, C_{M}$ are the eigenvalues and eigenvectors of the sample covariance matrix $\hat{R}$.

For the rest of the derivation, the covariance matrix will be defined as $R_{\jmath}=V D V^{\dagger}$ and the sample covariance matrix will be $\hat{R}=V \tilde{D} V^{\dagger}$, after completing the eigendecomposition. It is known that $\mathrm{D}$ and $\tilde{D}$ are diagonal matrices containing 
the eigenvalues and $\mathrm{V}$ and $V^{\dagger}$ are matrices containing the eigenvectors. It will also be assumed that $V^{\dagger} V=I$. Substituting the estimates from Equation 3.1.18 into the log-likelihood in Equation 3.1.16:

$$
\begin{aligned}
L\left(\hat{\Theta_{\jmath}}\right) & =-N \log \left[\operatorname{det}\left(V D V^{\dagger}\right)\right]-\operatorname{tr}\left(\left(V D^{-1} V^{\dagger}\right)\left(V \tilde{D} V^{\dagger}\right)\right) \\
& =-N \log \left[\operatorname{det}\left(V D V^{\dagger}\right)\right]-\operatorname{tr}\left(D^{-1} \tilde{D}\right)
\end{aligned}
$$

After some straightforward mathematical manipulations the log-likelihood function becomes:

$$
L\left(\hat{\Theta_{\jmath}}\right)=\log \left(\frac{\prod_{\imath=\jmath+1}^{M} l_{\imath}^{\frac{1}{M-\jmath}}}{\frac{1}{M-\jmath} \sum_{\imath=\jmath+1}^{M} l_{\imath}}\right)^{(M-\jmath) N}
$$

Substituting the log-likelihood into the equation for the AIC:

$$
A I C=-2 \log \left(\frac{\prod_{\imath=\jmath+1}^{M} l_{l}^{\frac{1}{M-\jmath}}}{\frac{1}{M-\jmath} \sum_{\imath=\jmath+1}^{M} l}\right)^{(M-\jmath) N}+2 p(2 M-p)
$$

Substituting the log-likelihood into the equation for the MDL:

$$
M D L=-\log \left(\frac{\prod_{\imath=\jmath+1}^{M} l_{l}^{\frac{1}{M-\jmath}}}{\frac{1}{M-\jmath} \sum_{l=\jmath+1}^{M} l_{l}}\right)^{(M-\jmath) N}+\frac{1}{2} p(2 M-p) \log N
$$

A consistent estimator would be expected to yield the true number of emitters with probability one as the sample size increases to infinity. In [20], the consistency of the MDL and AIC estimators is discussed in detail. It is shown that the MDL criterion provides a consistent estimate, while the AIC provides an inconsistent estimate that tends to overestimate the number of emitters. 
The formulation of the Emitter Number Estimation algorithm may be modified to suit the individual problem that it is being used to solve. In many situations, pulses are collected over long periods of time which would result in an extremely large covariance matrix if the pulse model that was introduced earlier in this section was used. This would cause the eigendecomposition of the covariance matrix to be an impractical calculation. In [25], the pulse signal model is adjusted to make the size of the covariance matrix dependent on the number of samples within the pulses rather than the number of pulses themselves. In [12], the K-means algorithm is used to initially cluster the pulses into $\tilde{M}$ clusters where $\tilde{M}$ should be much greater than the expected number of emitters. Using this technique, the covariance matrix would be reduced to a size of $\tilde{M} \times \tilde{M}$. 


\subsection{Pulse Comparator Algorithm}

The statistical model for the Pulse Comparator algorithm will be derived for two pulses with the assumption that they have the same underlying pulse shape or modulation. It is also assumed that the In-phase (I) and Quadrature (Q) components of the two received radar pulses are sampled at a sufficiently high sampling rate so that the pulses can be reconstructed with the digital samples. This can be achieved by following the Nyquist-Shannon Sampling Theorem which states that the sampling frequency must be greater than two times the bandwidth of the signal (i.e. $f_{s}>2 B$ ).

The following formulation is obtained using [21], and it describes two pulses that come from the same emitter but are misaligned in time and frequency. Both $\mathrm{x}$ and $\mathrm{y}$ are complex vectors each containing $\mathrm{N}$ samples. It is worth noting that the variables $\mathrm{x}$ and $\mathrm{y}$ do not relate to the variables that were defined for the Emitter Number Estimation algorithm derivation.

$$
\begin{aligned}
& x=\alpha T\left(\tau_{x}\right) \Omega\left(\omega_{x}\right) \mu+\epsilon_{x} \\
& y=\beta T\left(\tau_{y}\right) \Omega\left(\omega_{y}\right) \mu+\epsilon_{y}
\end{aligned}
$$

where:

- $\alpha=$ complex values representing the received amplitude and phase of the first pulse

- $\beta=$ complex values representing the received amplitude and phase of the second pulse 
- $T(\tau)=$ unitary matrix representing the time delay between the two pulses

- $\Omega(\omega)=$ unitary matrix representing the shift in frequency between the two pulses

- $\mu=$ unit vector with $\mathrm{N}$ complex elements representing the basic pulse profile

- $\epsilon=$ independent Gaussian distributed vector random variable

The $\epsilon$ satisfies $E(\epsilon)=0$ and $E\left(\epsilon \epsilon^{H}\right)=2 \sigma^{2} I$ where $\sigma$ is the noise variance and $\mathrm{I}$ is the $\mathrm{N} \times \mathrm{N}$ identity matrix. To simplify the formulation, let $\epsilon_{x}=\epsilon_{y}=\epsilon$.

The pulses may be aligned in time by using cross-correlation. Once aligned, the $T(\tau)$ can be set to one and Equations 3.2.1 and 3.2.2 become:

$$
\begin{aligned}
& x=\alpha \Omega\left(\omega_{x}\right) \mu+\epsilon_{x} \\
& y=\beta \Omega\left(\omega_{y}\right) \mu+\epsilon_{y}
\end{aligned}
$$

Rewriting equations 3.2 .3 and 3.2.4:

$$
\begin{aligned}
& \tilde{x}=\alpha \mu+\epsilon \\
& \tilde{y}=\beta \mu+\epsilon
\end{aligned}
$$

where:

$$
\begin{aligned}
& \tilde{x}=\Omega^{-1}\left(\omega_{x}\right) x \\
& \tilde{y}=\Omega^{-1}\left(\omega_{y}\right) y
\end{aligned}
$$

The probability functions for $\tilde{x}$ and $\tilde{y}$ at a given $(\mu, \alpha)$ and $(\mu, \beta)$ are defined to be:

$$
\begin{aligned}
& P_{\tilde{x}}(\tilde{x} \mid \mu, \alpha) \\
& P_{\tilde{y}}(\tilde{y} \mid \mu, \beta)
\end{aligned}
$$


The probability functions of the Gaussian complex random vectors $\tilde{x}$ and $\tilde{y}$ with $\mathrm{N}$ independent observations can be represented as a normal distribution as follows:

$$
\begin{aligned}
P_{\tilde{x}}(\tilde{x} \mid \mu, \alpha) & =\frac{1}{(\sigma \sqrt{2 \pi})^{N}} \exp \left[-\frac{1}{2 \sigma^{2}}\|\tilde{x}-\alpha \mu\|^{2}\right] \\
P_{\tilde{y}}(\tilde{y} \mid \mu, \beta) & =\frac{1}{(\sigma \sqrt{2 \pi})^{N}} \exp \left[-\frac{1}{2 \sigma^{2}}\|\tilde{y}-\beta \mu\|^{2}\right]
\end{aligned}
$$

The joint probability of $P_{\tilde{x}}$ and $P_{\tilde{y}}$ is given by:

$$
\begin{aligned}
P(\tilde{x}, \tilde{y}) & =P_{\tilde{x}}(\tilde{x} \mid \mu, \alpha) P_{\tilde{y}}(\tilde{y} \mid \mu, \beta) \\
& =P(\tilde{x}, \tilde{y} \mid \mu, \alpha, \beta) \\
& =\frac{1}{\left(2 \pi \sigma^{2}\right)^{N}} \exp \left[-\frac{1}{2 \sigma^{2}}\left(\|\tilde{x}-\alpha \mu\|^{2}+\|\tilde{y}-\beta \mu\|^{2}\right)\right]
\end{aligned}
$$

The joint likelihood function is identical to the joint probability function in Equation 3.2.13. In order to obtain the maximum likelihood, the joint likelihood function needs to be maximized with respect to $\mu, \alpha$ and $\beta$ as follows:

$$
\begin{aligned}
\max _{\mu, \alpha, \beta} L(\mu, \alpha, \beta \mid \tilde{x}, \tilde{y}) & =\max _{\mu, \alpha, \beta} \frac{1}{\left(2 \pi \sigma^{2}\right)^{N}} \exp \left[-\frac{1}{2 \sigma^{2}}\left(\|\tilde{x}-\alpha \mu\|^{2}+\|\tilde{y}-\beta \mu\|^{2}\right)\right] \\
& =\max _{\mu, \alpha, \beta} \frac{1}{\left(2 \pi \sigma^{2}\right)^{N}} \exp \left(-\frac{1}{2 \sigma^{2}} R\right)
\end{aligned}
$$

where:

$$
\begin{aligned}
R & =\|\tilde{x}-\alpha \mu\|^{2}+\|\tilde{y}-\beta \mu\|^{2} \\
& =R_{\tilde{x}}(\alpha, \mu)+R_{\tilde{y}}(\beta, \mu)
\end{aligned}
$$

In order to maximize the joint likelihood function, the term in Equation 3.2.16 should be minimized. First, the $R_{\tilde{x}}(\alpha, \mu)$ term is minimized and the other term is 
minimized later in a similar fashion:

$$
\begin{aligned}
R_{\tilde{x}}(\alpha, \mu) & =\|\tilde{x}-\alpha \mu\|^{2} \\
& =(\tilde{x}-\alpha \mu)^{\dagger}(\tilde{x}-\alpha \mu) \\
& =\tilde{x}^{\dagger} \tilde{x}-\tilde{x}^{\dagger} \alpha \mu-\alpha^{*} \mu^{\dagger} \tilde{x}+|\alpha|^{2} \mu^{\dagger} \mu \text { (Note: } \mu^{\dagger} \mu=1 \text { since } \mu \text { is a unit vector) } \\
& =\tilde{x}^{\dagger} \tilde{x}-\tilde{x}^{\dagger} \alpha \mu-\alpha^{*} \mu^{\dagger} \tilde{x}+\alpha^{*} \alpha \\
& =\tilde{x}^{\dagger} \tilde{x}-\tilde{x}^{\dagger} \alpha \mu+\alpha^{*}\left(\alpha-\mu^{\dagger} \tilde{x}\right)+\left(\tilde{x}^{\dagger} \mu\right)^{*}\left(\tilde{x}^{\dagger} \mu\right)-\left|\tilde{x}^{\dagger} \mu\right|^{2} \\
& =\alpha^{*}\left(\alpha-\mu^{\dagger} \tilde{x}\right)-\tilde{x}^{\dagger} \mu\left(\alpha-\mu^{\dagger} \tilde{x}\right)+\tilde{x}^{\dagger} \tilde{x}-\left|\tilde{x}^{\dagger} \mu\right|^{2} \\
& =\left(\alpha^{*}-\tilde{x}^{\dagger} \mu\right)\left(\alpha-\mu^{\dagger} \tilde{x}\right)+\tilde{x}^{\dagger} \tilde{x}-\left|\tilde{x}^{\dagger} \mu\right|^{2} \\
& =\left(\alpha-\mu^{\dagger} \tilde{x}\right)^{*}\left(\alpha-\mu^{\dagger} \tilde{x}\right)+\tilde{x}^{\dagger} \tilde{x}-\left|\tilde{x}^{\dagger} \mu\right|^{2} \\
& =\left\|\alpha-\mu^{\dagger} \tilde{x}\right\|+\tilde{x}^{\dagger} \tilde{x}-\left|\tilde{x}^{\dagger} \mu\right|^{2}
\end{aligned}
$$

Equation 3.2 .17 can be minimized by setting $\alpha=\mu^{\dagger} \tilde{x}$ :

$$
R_{\tilde{x}}(\alpha, \mu)=\tilde{x}^{\dagger} \tilde{x}-\left|\tilde{x}^{\dagger} \mu\right|^{2}
$$

Similarly,

$$
R_{\tilde{y}}(\beta, \mu)=\tilde{y}^{\dagger} \tilde{y}-\left|\tilde{y}^{\dagger} \mu\right|^{2}
$$

Substituting Equations 3.2.18 and 3.2.19 into Equation 3.2.16:

$$
\begin{aligned}
R & =\tilde{x}^{\dagger} \tilde{x}-\left|\tilde{x}^{\dagger} \mu\right|^{2}+\tilde{y}^{\dagger} \tilde{y}-\left|\tilde{y}^{\dagger} \mu\right|^{2} \\
& =\tilde{x}^{\dagger} \tilde{x}+\tilde{y}^{\dagger} \tilde{y}-\left(\mu^{\dagger} \tilde{x}\right)\left(\tilde{x}^{\dagger} \mu\right)-\left(\mu^{\dagger} \tilde{y}\right)\left(\tilde{y}^{\dagger} \mu\right) \\
& =\tilde{x}^{\dagger} \tilde{x}+\tilde{y}^{\dagger} \tilde{y}-\mu^{\dagger}\left(\tilde{x} \tilde{x}^{\dagger}+\tilde{y} \tilde{y}^{\dagger}\right) \mu
\end{aligned}
$$

Equation 3.2.20 is minimized by maximizing the $\mu^{\dagger}\left(\tilde{x} \tilde{x}^{\dagger}+\tilde{y} \tilde{y}^{\dagger}\right) \mu$ term. This term can be maximized by choosing the $\mu$ value to be the eigenvector of $\tilde{x} \tilde{x}^{\dagger}+\tilde{y} \tilde{y}^{\dagger}$ that 
has the largest associated eigenvalue. It would have the same eigenvalues as the following Hermitian matrix:

$$
\left(\begin{array}{cc}
\tilde{x}^{\dagger} \tilde{x} & \tilde{x}^{\dagger} \tilde{y} \\
\tilde{y}^{\dagger} \tilde{x} & \tilde{y}^{\dagger} \tilde{y}
\end{array}\right)
$$

Setting $A=\tilde{x}^{\dagger} \tilde{x}, B=\tilde{y}^{\dagger} \tilde{x}$ and $C=\tilde{y}^{\dagger} \tilde{y}$ the eigenvalues can be obtained:

$$
\left(\begin{array}{cc}
A & B^{\dagger} \\
B & C
\end{array}\right) \mathrm{v}=\lambda \mathrm{v}
$$

Solving the equation above:

$$
\begin{gathered}
(A-\lambda)(C-\lambda)-|B|^{2}=0 \\
\lambda^{2}-(A+C) \lambda+A C-|B|^{2}=0 \\
\lambda=\frac{A+C \pm \sqrt{(A+C)^{2}-4 A C+4|B|^{2}}}{2}
\end{gathered}
$$

Therefore, we subtract the largest eigenvalue as follows:

$$
\begin{aligned}
R & =A+C-\frac{1}{2}\left[A+C+\sqrt{(A+C)^{2}-4 A C+4|B|^{2}}\right. \\
& =A+C-\frac{1}{2}\left[A+C+\sqrt{(A-C)^{2}+4|B|^{2}}\right. \\
& =\frac{1}{2}[A+C]-\frac{1}{2} \sqrt{(A-C)^{2}+4|B|^{2}}
\end{aligned}
$$

Substituting back in for $\mathrm{A}, \mathrm{B}$, and $\mathrm{C}$ the final equation is:

$$
R=\frac{1}{2}\left[\tilde{x}^{\dagger} \tilde{x}+\tilde{y}^{\dagger} \tilde{y}\right]-\frac{1}{2} \sqrt{\left(\tilde{x}^{\dagger} \tilde{x}-\tilde{y}^{\dagger} \tilde{y}\right)^{2}+4\left|\tilde{y}^{\dagger} \tilde{x}\right|^{2}}
$$

Therefore, the final equation for the maximum likelihood function is:

$$
\max _{\mu, \alpha, \beta} L(\mu, \alpha, \beta \mid \tilde{x}, \tilde{y})=\max _{\mu, \alpha, \beta} \frac{1}{\left(2 \pi \sigma^{2}\right)^{N}} \exp \left(-\frac{1}{2 \sigma^{2}} R\right)
$$

Where:

$$
R=\frac{1}{2}\left[\tilde{x}^{\dagger} \tilde{x}+\tilde{y}^{\dagger} \tilde{y}\right]-\frac{1}{2} \sqrt{\left(\tilde{x}^{\dagger} \tilde{x}-\tilde{y}^{\dagger} \tilde{y}\right)^{2}+4\left|\tilde{y}^{\dagger} \tilde{x}\right|^{2}}
$$


And:

$$
0 \leq R \leq \min \left(\tilde{x}^{\dagger} \tilde{x}, \tilde{y}^{\dagger} \tilde{y}\right)
$$

The terms $\tilde{x}^{\dagger} \tilde{x}$ and $\tilde{y}^{\dagger} \tilde{y}$ represent the energies of the individual pulses and are not dependent on the phase or the frequency modulations on each pulse, while the term $\tilde{y}^{\dagger} \tilde{x}$ does depend on the modulations present within each pulse. Therefore, the $\mathrm{R}$ term depends on modulations through the term $4\left|\tilde{y}^{\dagger} \tilde{x}\right|^{2}$. Analyzing the equation, it is clear that if the pulses are exactly the same the $\mathrm{R}$ term will be equal to zero. Conversely, if the pulses are different then the $\mathrm{R}$ term will be approximately equal to the energy of one of the pulses.

\section{Chapter Summary}

This chapter provided a detailed derivation for both the Emitter Number Estimation algorithm and the Pulse Comparator algorithm. The Emitter Number Estimation algorithm uses the Information Theoretic Criteria in the form of the AIC or MDL equation. The formula for the Pulse Comparator algorithm is obtained by maximizing the joint likelihood function for two pulses that are assumed to contain the same underlying pulse shape. The next chapter will illustrate how these algorithms may be used to create an offline solution and an online solution. 


\section{Chapter 4}

\section{Proposed Solutions}

This thesis proposes an offline solution that is capable of deinterleaving a large number of pulses. The offline solution consists of a three stage procedure. First, the pulses are sorted by their interpulse parameters, and then the next two stages use intrapulse parameters to obtain a more refined grouping result. Following the offline solution, an online solution will be outlined that is able to sort pulses in real-time as they are received. The online solution evaluates the similarity between pulses using their intrapulse characteristics. 


\section{Offline Solution}

An offline solution requires that a large number of pulses be available for the initial classification [9]. The offline solution in this thesis consists of three main stages, which are illustrated in Figure 4.1. Each stage will be discussed in more detail.

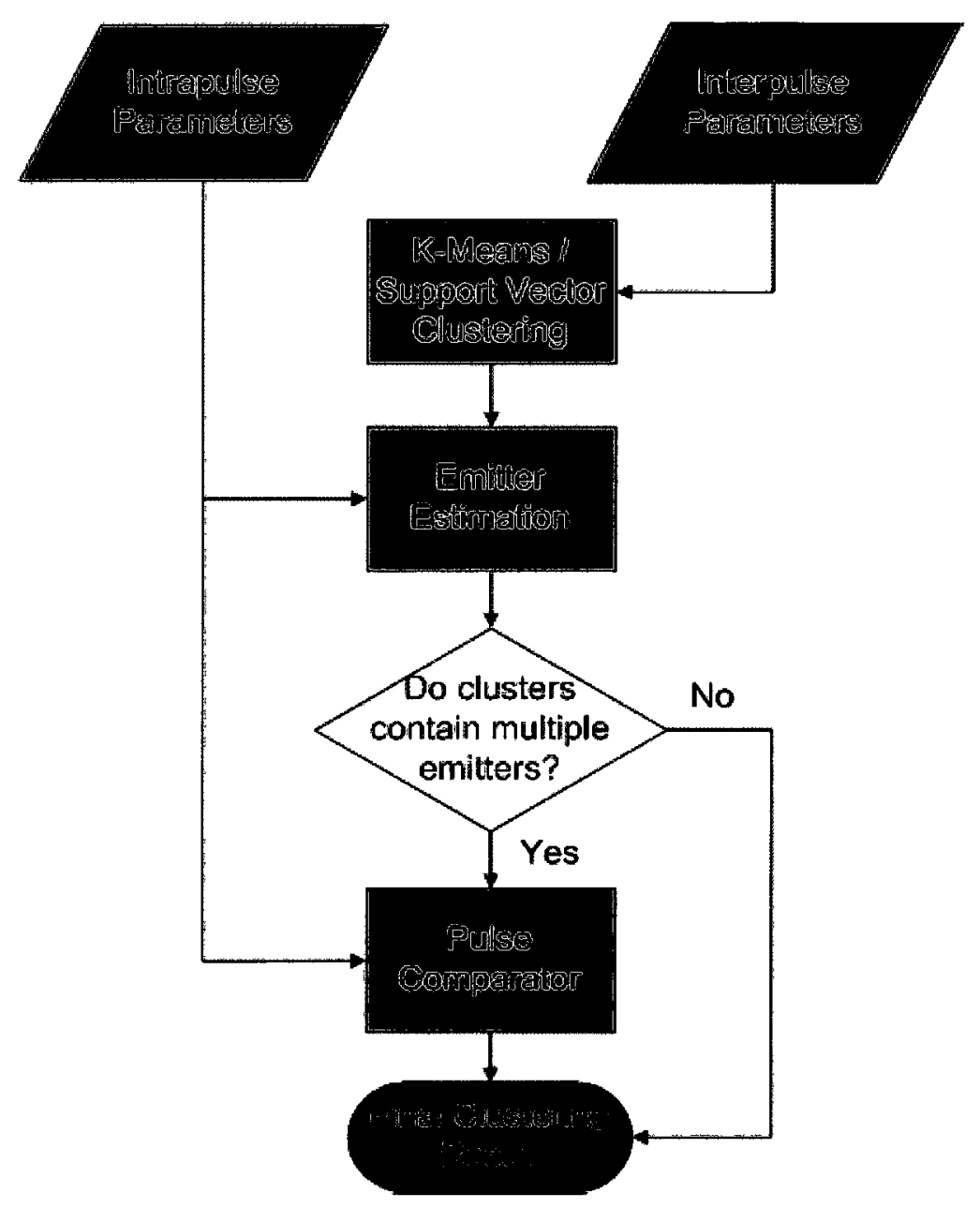

Figure 4.1: Offline Solution 


\section{Stage 1:}

Initially, interpulse parameters such as Radio Frequency (RF), Pulse Width (PW), Time of Arrival (TOA), Direction of Arrival (DOA), and Pulse Repetition Interval (PRI) are used to perform the clustering operation. For this stage, many different unsupervised learning algorithms may be used. For simplicity, the well-known K-Means algorithm was chosen to perform the sorting. One disadvantage of using the K-Means algorithm is that the number of clusters, $\mathrm{K}$, must be specified by the user. The value for the initial number of clusters can either be chosen randomly or based on intuition, if the user has a more efficient way to approximate the number of emitters in the surrounding environment. In order to remove the requirement for user-defined inputs, the method that is described in [16] may be implemented. Using a subset of the overall data set, Support Vector Clustering (SVC) along with Type-Entropy Pattern Recognition are used to estimate the number of clusters and their centroids. The K-Means algorithm then uses this initial estimate to sort the full data set. Once the cluster analysis in Stage 1 is complete, it is assumed that there are at least $\mathrm{k}$ different emitters existing within the data. Figure 4.2 shows the steps that are involved in this stage of the offline solution. 


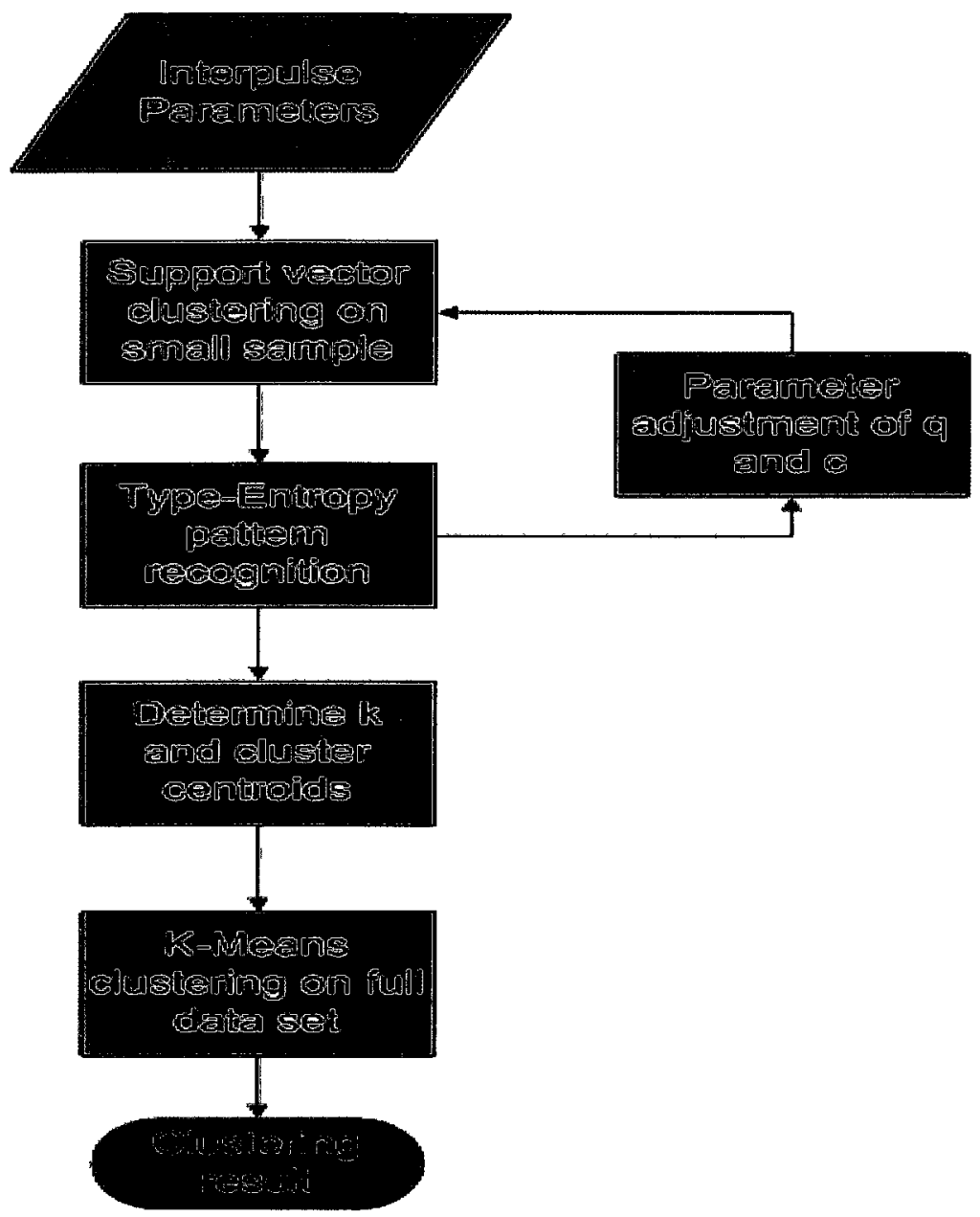

Figure 4.2: Stage 1 Of The Offline Solution 


\section{Stage 2:}

Following the initial sorting stage, the resulting clusters must be examined to confirm whether any of them require further analysis. Stage 2 will be completed by using the Emitter Number Estimation algorithm that was introduced in the previous chapter. Rather than using the interpulse parameters, this algorithm will use the intrapulse characteristics in the form of the individual pulse sample In-phase (I) and Quadrature (Q) data. If the Emitter Number Estimation technique finds that each cluster contains pulses that originated from one single emitter, then the clustering result from Stage 1 is maintained as the final solution. On the other hand, if any of the clusters are found to have pulses from multiple sources then these clusters are advanced to the next stage. Figure 4.3 illustrates the operation of the Emitter Number Estimation stage. 


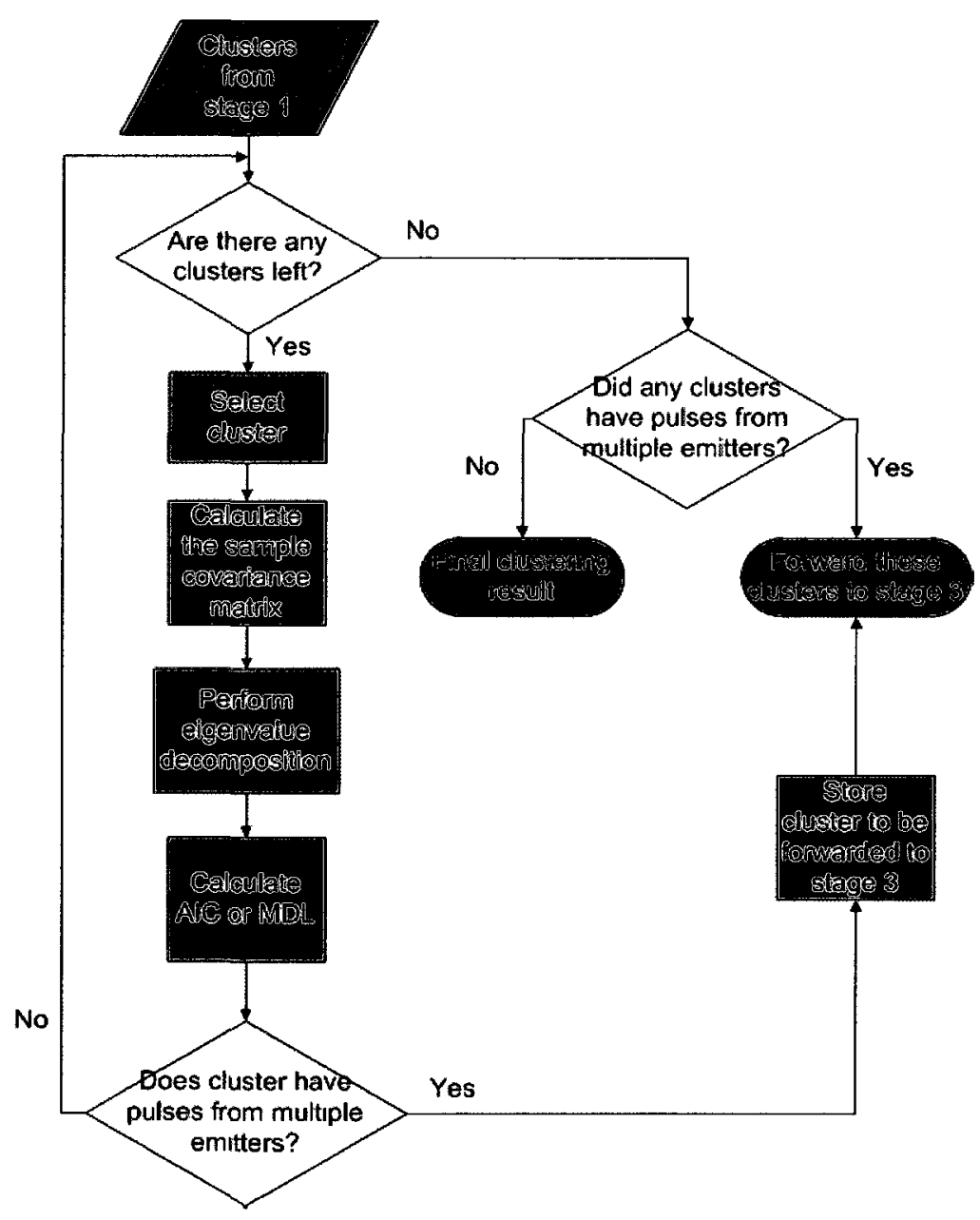

Figure 4.3: Stage 2 Of The Offline Solution 


\section{Stage 3:}

Any clusters that are expected to contain pulses from multiple emitters are flagged in Stage 2 and must be clustered further to obtain a more optimal grouping result. In this stage, the pulse sorting is completed by using the Pulse Comparator algorithm that was introduced in the previous chapter. In order to successfully sort the pulses, the appropriate threshold for pulse similarity must be selected. This threshold must be selected by the user and depends on the expected minimum SNR for the pulses that are received. Following the threshold selection, the rest of the sorting procedure will be performed as described in Figure 4.4. The algorithm begins by selecting two pulses from the cluster and calculating whether these pulses came from the same emitter using the decision-criteria that was defined previously. Next, these pulses are either split into two different clusters or they are combined into one cluster. Each cluster is represented by the mean of the pulses that are within it. Therefore, the mean of each cluster is updated every time a pulse is added to it. After this initial step, all successive pulses are compared to the mean values of the existing clusters. If they are not similar to any clusters, then a new cluster is initiated. Following this stage, the resulting cluster arrangement should provide an accurate representation of the emitters in the surrounding environment. 


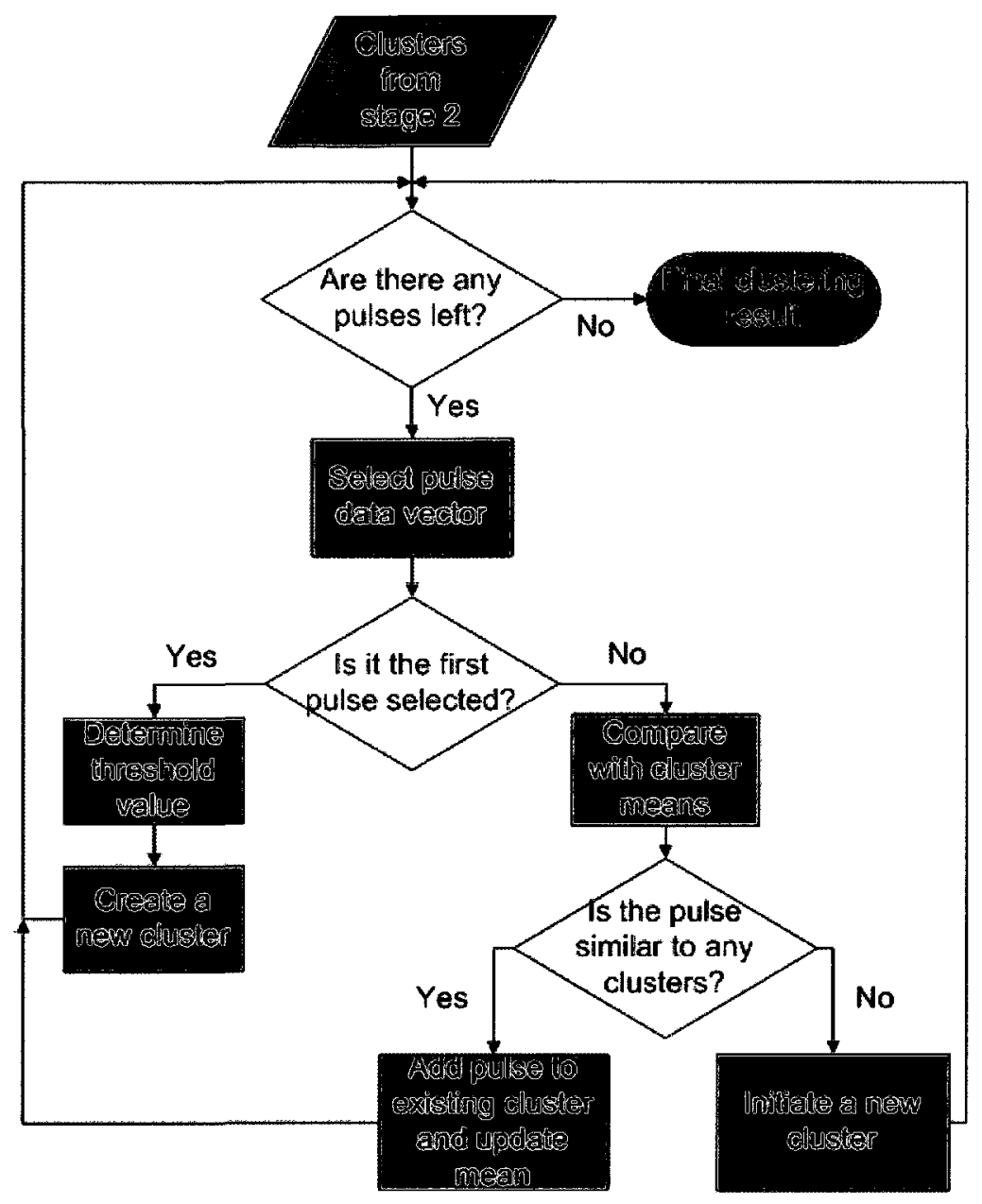

Figure 4.4: Stage 3 Of The Offline Solution 


\section{Online Solution}

Online algorithms are required in ES applications to provide the capability of making quick decisions. An online algorithm should be able to update the cluster structure every time a new pulse arrives [9]. The third stage of the offline solution that outlined the sorting technique using the Pulse Comparator is proposed as a possible online solution. The operation of the Pulse Comparator is described in Figure 4.4 above. The only difference between the third stage of the offline solution and the online solution is the removal of the feedback loop. This loop is not required since the pulses are forwarded as they arrive at the receiver. Along with the advantage of updating the cluster structure each time a pulse is received, this algorithm also reduces the requirement for data storage because only the cluster means are required to be stored. The resulting online solution is shown in Figure 4.5. 


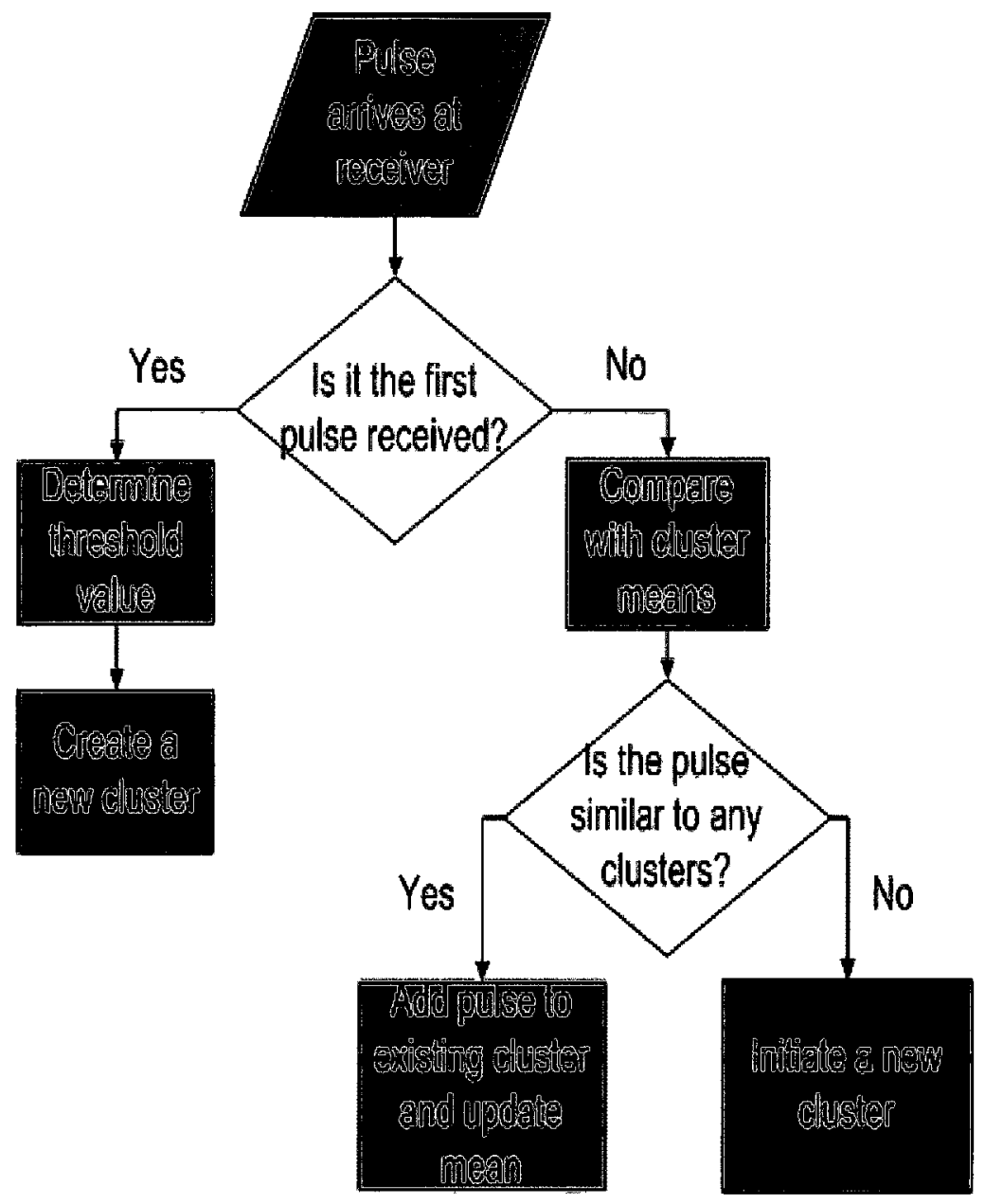

Figure 4.5: Online Solution 


\section{Chapter Summary}

This chapter described an offline solution and an online solution that may be used for deinterleaving a set of pulses. The offline solution is able to sort a large number of pulses using a three stage process. The first stage consists of an interpulse sorting technique that combines the use of the K-Means algorithm with the Support Vector Clustering method. Then, the second stage uses the Emitter Number Estimation algorithm to verify whether the clusters contained pulses from one emitter or multiple emitters. Finally, the third stage uses the Pulse Comparator to refine the initial clustering. The next chapter will define a simulated scenario that will subsequently be used to test the algorithms for the offline and online solutions. 


\section{Chapter 5}

\section{Results}

In this chapter, the offline and online solutions that were previously introduced will be tested to show their validity. Prior to outlining the results of the testing, a simulated scenario will be introduced. Using this simulated scenario, each stage of the offline solution will be discussed and tested individually. Then, it will be shown that the third stage of the offline solution can be used as an online solution to sort pulses in real-time as they are received.

To provide context into the problem of pulse deinterleaving, a simulated scenario is illustrated in Figure 5.1. First, the problem depends on whether the EW equipment is being used strictly for long-term data gathering, or if it is used for real-time situational awareness. An ELINT receiver is typically used for long-term initiatives while an ES receiver is used to monitor potential immediate threats. The simulated scenario contains either an ELINT receiver or an ES receiver at the center of a coordinate system surrounded by a number of other radar systems. The offline solution will be applied in the case where the ELINT receiver is in the center, and 
the online solution will be applied when the ES receiver is centered. As part of the scenario, each radar system is assigned both interpulse and intrapulse characteristics. The interpulse parameters for each radar are chosen using typical figures from fielded systems, and they are shown in Table 5.1. For the analysis in this thesis, it will be assumed that the radar systems do not have the ability to perform frequency agility. It will also be assumed that the four tracking radars are close enough in proximity that the ELINT and ES receivers are not able to differentiate between their direction of arrıvals.

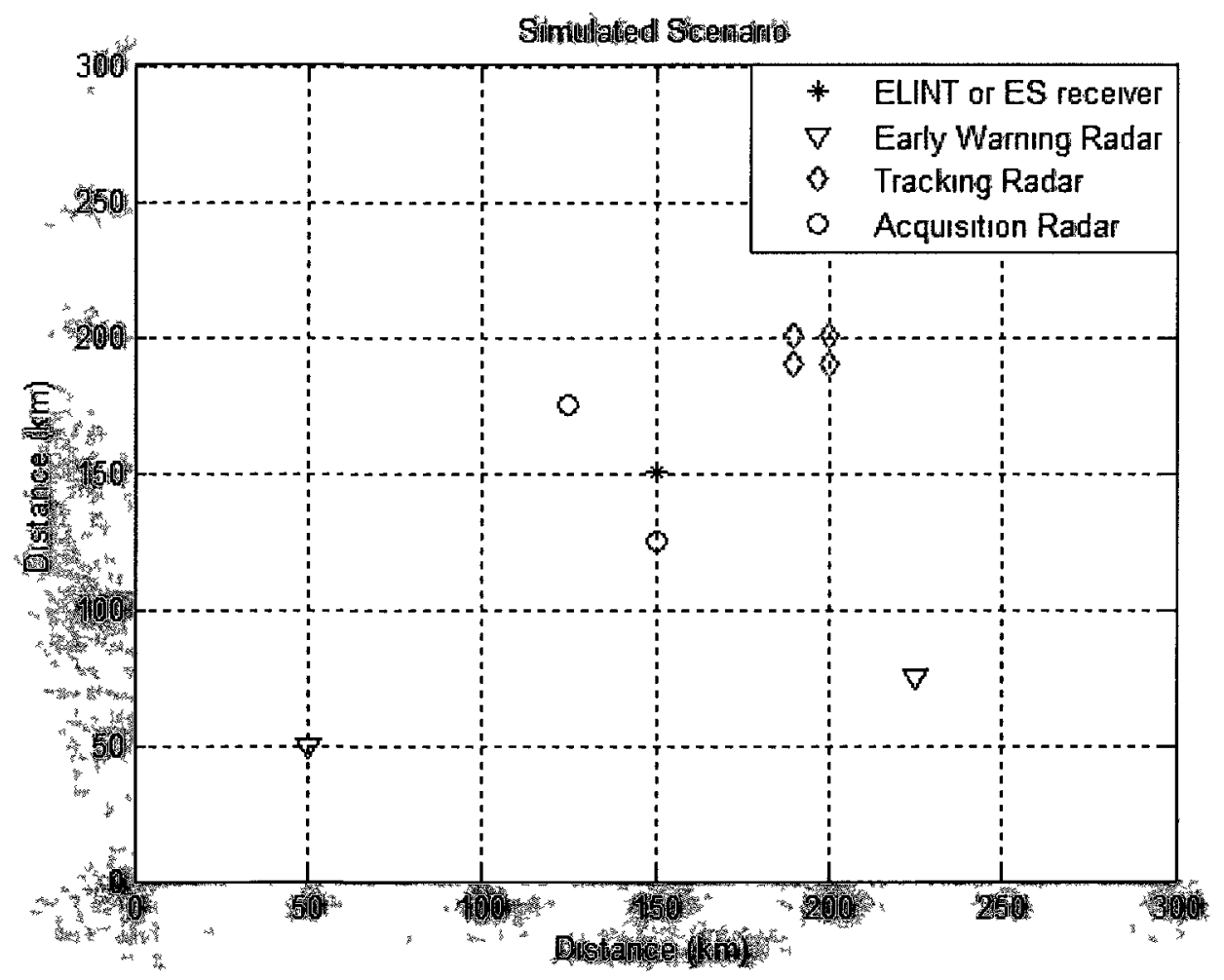

Figure 5.1: Simulated Scenario 


\begin{tabular}{|l|c|c|c|c|c|}
\hline & \multicolumn{2}{|l|}{$\begin{array}{l}\text { Early } \\
\text { Warning } \\
\text { Radars }\end{array}$} & \multicolumn{2}{l|l}{$\begin{array}{l}\text { Acquisition } \\
\text { Radars }\end{array}$} & Tracking Radars \\
\hline Number of the radar & 1 & 2 & 3 & 4 & $5,6,7,8$ \\
\hline PW (us) & 6 & 10 & 50 & 100 & 6 \\
\hline Frequency (GHz) & 1.3 & 1.7 & 2.7 & 3.2 & 10 \\
\hline Direction of Arrival (degrees) & 225 & 135 & 180 & 315 & 45 \\
\hline
\end{tabular}

Table 5.1: Interpulse Parameters for Radar Systems in the Simulated Scenario

The software tool used for the algorithm implementations and simulations was chosen to be Matrix Laboratory (MATLAB). MATLAB is a high-level language that allows the user to perform computationally intensive tasks quickly and efficiently. It is capable of handling large data sets and complex matrix operations [15]. MATLAB also includes a user-friendly interactive environment. It is commonly used for problem-solving within fields such as signal processing, statistics, pattern classification, and many more.

The pulses that were used for the experiments in this thesis were constructed with a pulse generator that was programmed within the MATLAB environment. Each radar system in the simulated scenario was assigned twenty pulses for the simulations. The pulse generator had a number of selectable interpulse and intrapulse parameters. Along with the various parameters, the Signal-to-Noise Ratio (SNR) could also be adjusted. The SNR is a very important factor to consider when analyzing the performance of an algorithm. In practice, received signals are never identical to the original transmission since they are always corrupted by noise. The effectiveness of the algorithms in this thesis is dependent on the SNR that is available within the received pulses. The effects of varying the SNR will be illustrated in the simulation results for the algorithms. The SNR will be defined 
using the following formula:

$$
S N R_{d B}=10 \log _{10}\left(\frac{A_{\text {signal }}}{A_{\text {norse }}}\right)^{2}=20 \log _{10}\left(\frac{A_{\text {signal }}}{A_{\text {norse }}}\right)
$$

Where $A_{\text {signal }}$ is the root mean square voltage of the signal and $A_{\text {norse }}$ is the root mean square voltage of the noise.

Since there are a variety of different interpulse parameters, most algorithms will use a subset to complete the pulse deinterleaving procedure. The first stage of the offline solution combines the use of SVC and K-Means to perform the pulse sorting operation. This stage uses a three-dimensional feature vector of the form $v_{\imath}=\left(P W_{\imath}, R F_{\imath}, D O A_{\imath}\right)$ where $\mathrm{i}$ is the pulse number. It can be observed that the four tracking radars have identical interpulse parameters, and they would appear to originate from one single target. The clustering result after the first stage of the offline solution is shown in Figure 5.2. Following the first stage of deinterleaving, the pulses were separated into five distinct groups. It is clear that this segmentation is not sufficient to provide an effective model of the surroundings. In order to be able to distinguish between the tracking radars, the intrapulse parameters will be used for the remaining segment of the proposed offline solution.

The intrapulse information will be represented with a set of complex samples. These samples can be used to obtain insight on the type of modulation profile within each pulse. There are a variety of pulse modulations available to a radar system designer when selecting which waveforms to utilize in their particular design. Within the EW field, there are two different types of pulse modulations that may be observed: Intentional Modulation On Pulse (IMOP) and Unintentional Modulation On Pulse (UMOP). Both types contain important information that can 


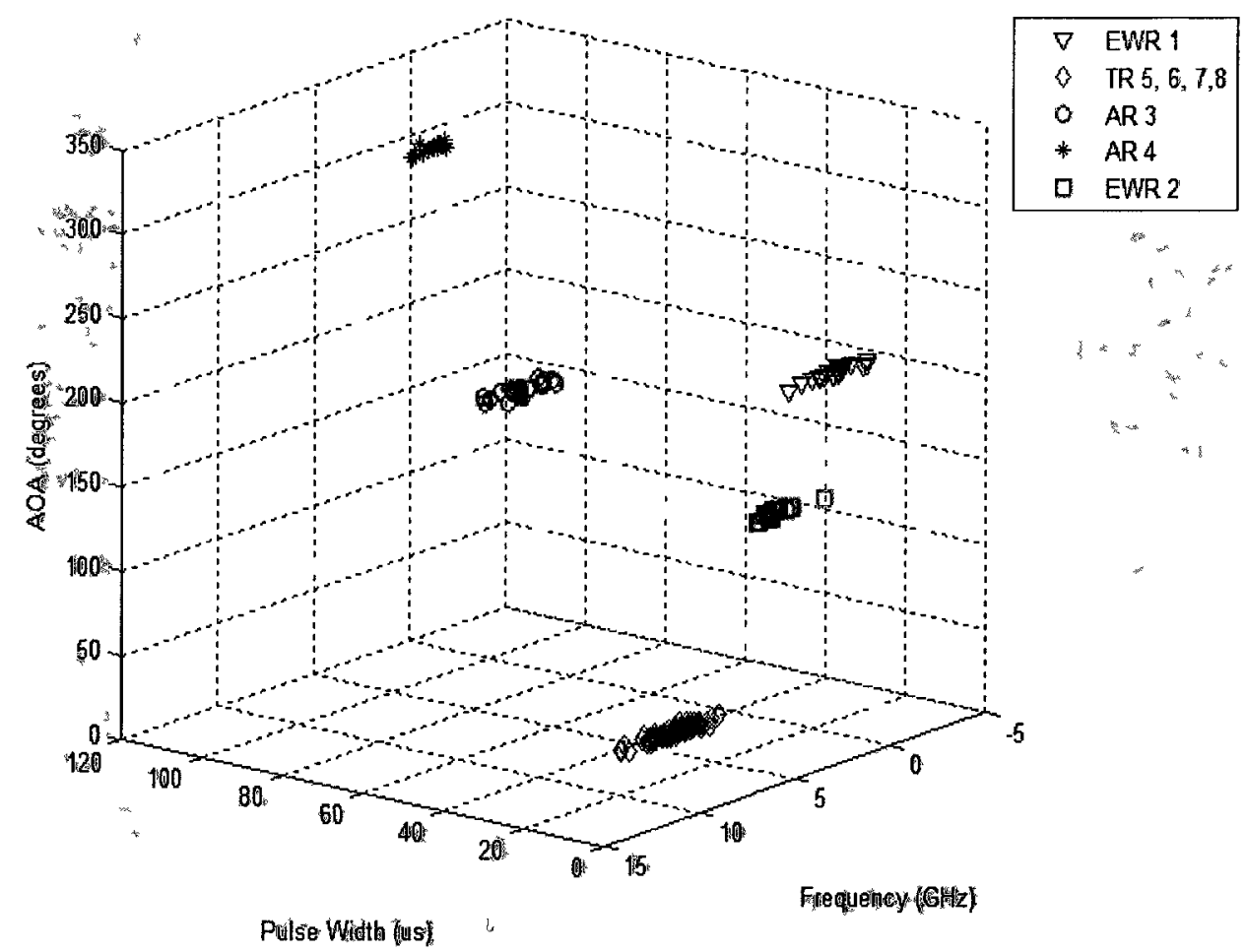

Figure 5.2: Deinterleaving Using The Interpulse Parameters

be used for pulse deinterleaving and emitter identification [21]. This thesis will focus on utilizing IMOP characteristics rather than UMOP characteristics to perform the sorting for the remaining stages of the proposed offline solution. A waveform with no modulation is illustrated in Figure 5.3 and some typical IMOP waveforms are illustrated in Figures 5.4, 5.5 and 5.6. The waveforms in these figures contain a SNR of $21 \mathrm{~dB}$. Figure 5.4 shows a Linear Frequency Modulated (LFM) pulse or chirp pulse which has the frequency increasing in a linear fashion by about $10 \mathrm{MHz}$. Along with the frequency profile, the I and Q components and the phase profile are also displayed. In Figure 5.5, a Frequency-Shift Keying (FSK) modulation scheme is used where the frequency changes discretely in the following order: $-5 \mathrm{MHz}, 10 \mathrm{MHz}$, 
$0 \mathrm{MHz},-10 \mathrm{MHz}, 0 \mathrm{MHz},-10 \mathrm{MHz}$. Figure 5.6 shows a form of Phase-Shift Keying that is known as 13-Bit Barker Code modulation. This is a biphase modulation scheme which uses 180 degree changes in phase. In Figure 5.6, the phase changes in the following order: $0,180,0,180,0,0,180,0,0,180,0,180,0$. To illustrate how variations in the SNR affect the pulse shape, Appendix B contains three figures that show the LFM pulse with SNR ratios of $13 \mathrm{~dB}, 7 \mathrm{~dB}$ and $1 \mathrm{~dB}$, respectively. 


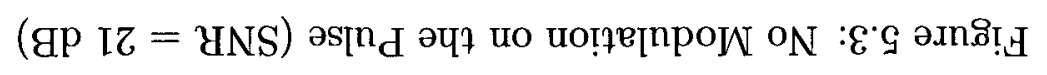
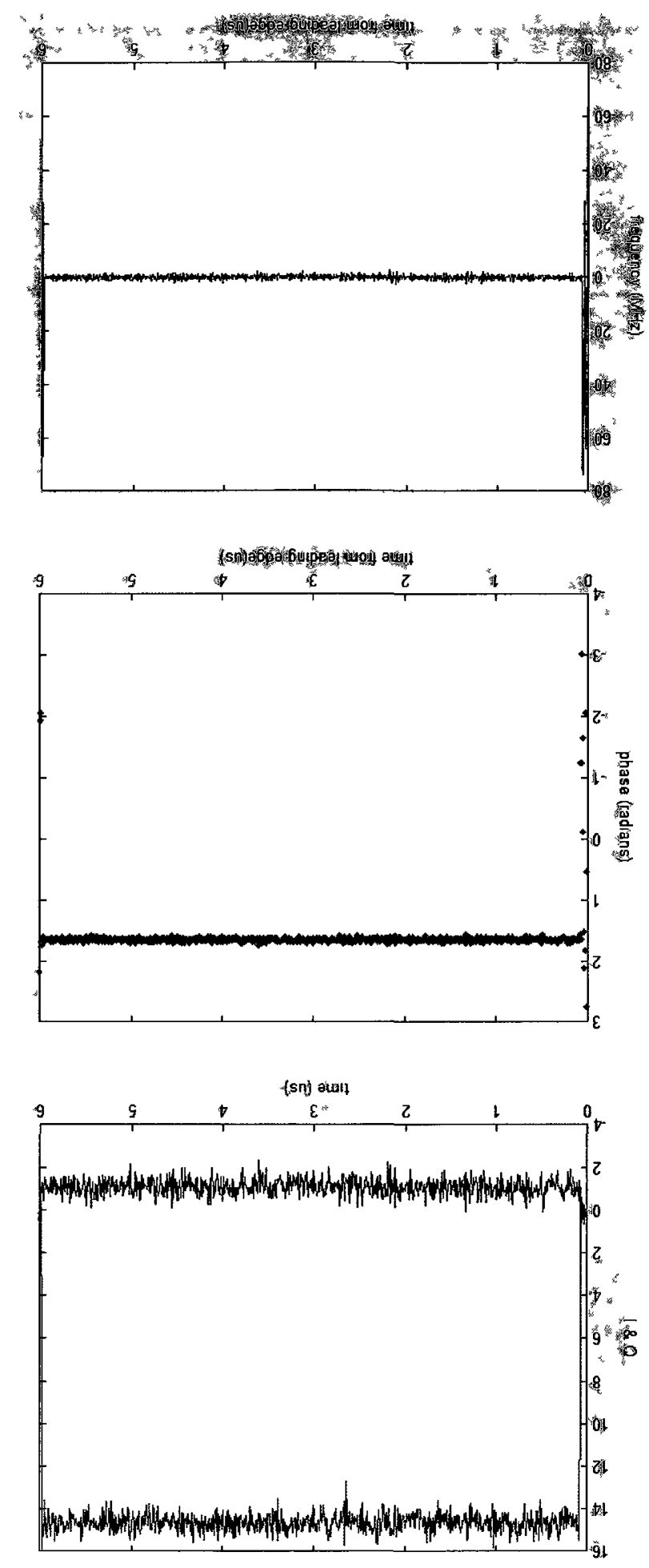

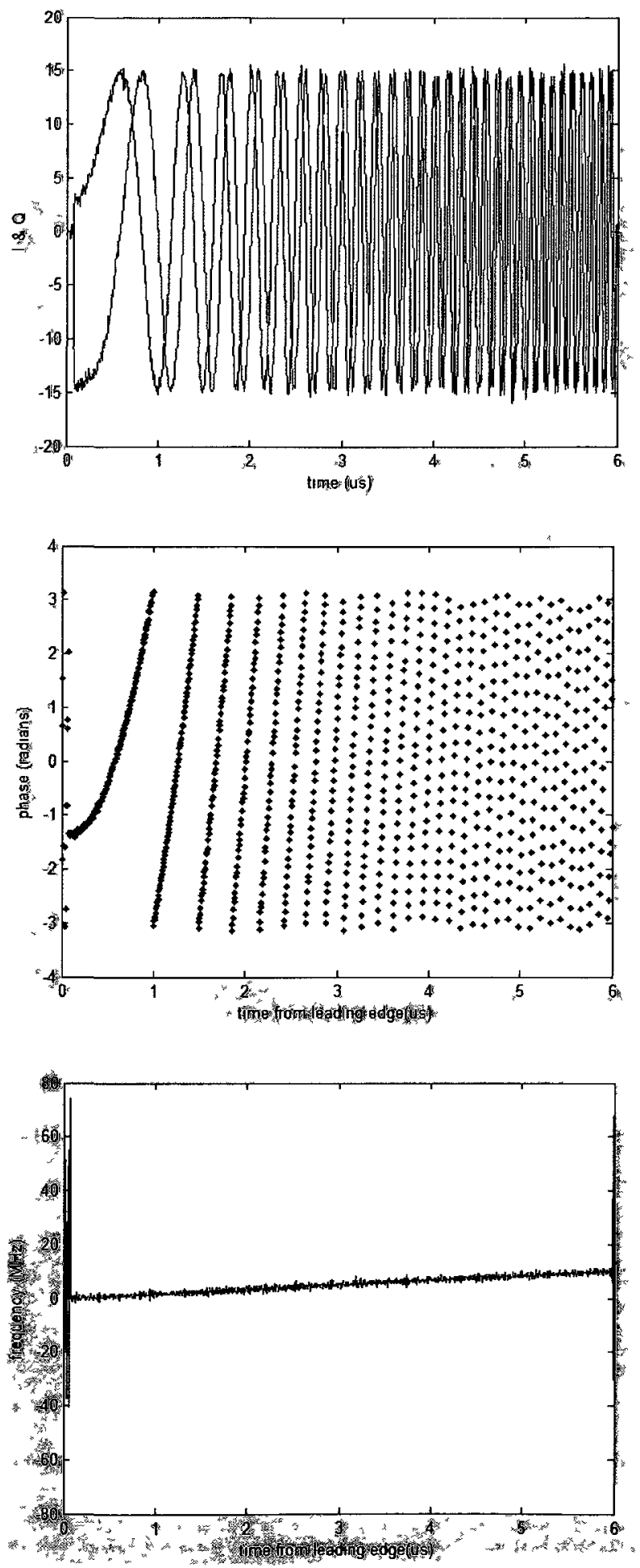

Figure 5.4: Linear Frequency Modulation $(\mathrm{LFM})(\mathrm{SNR}=21 \mathrm{~dB})$ 

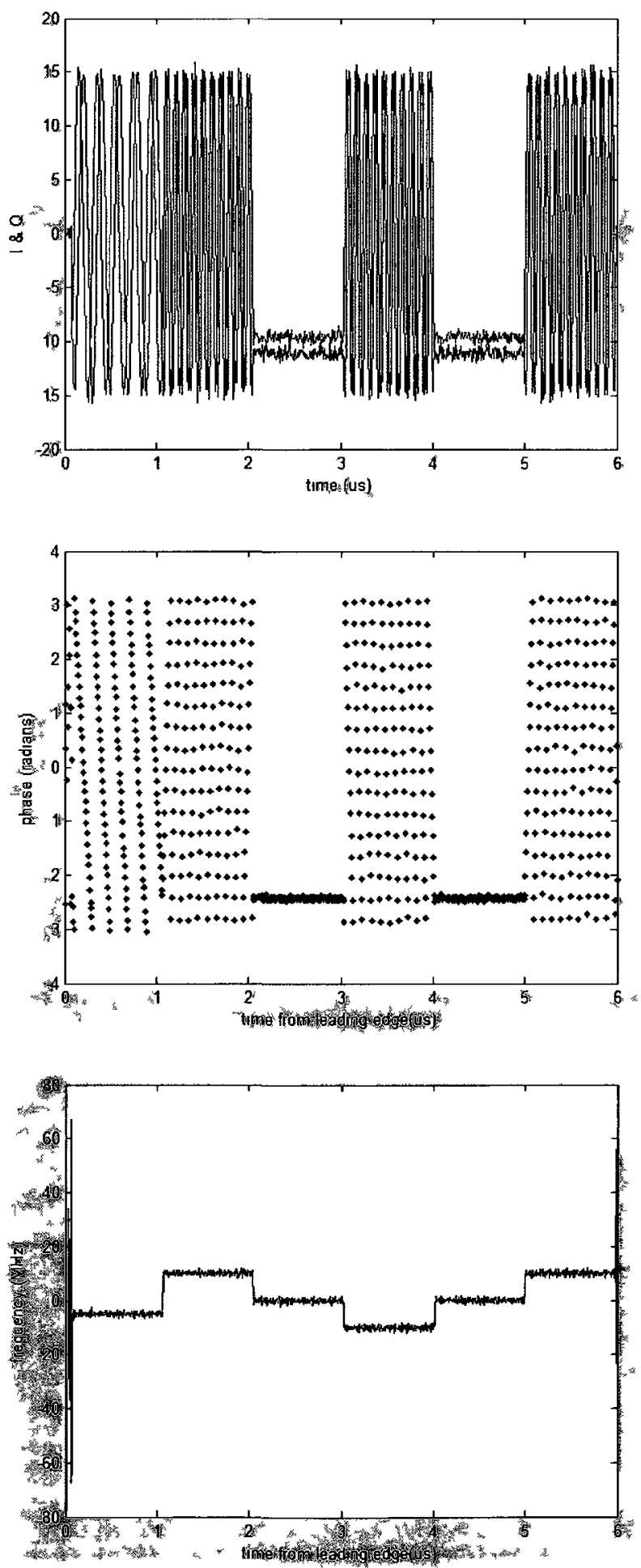

Figure 5.5: Frequency-Shift Keying (FSK) $(\mathrm{SNR}=21 \mathrm{~dB})$ 

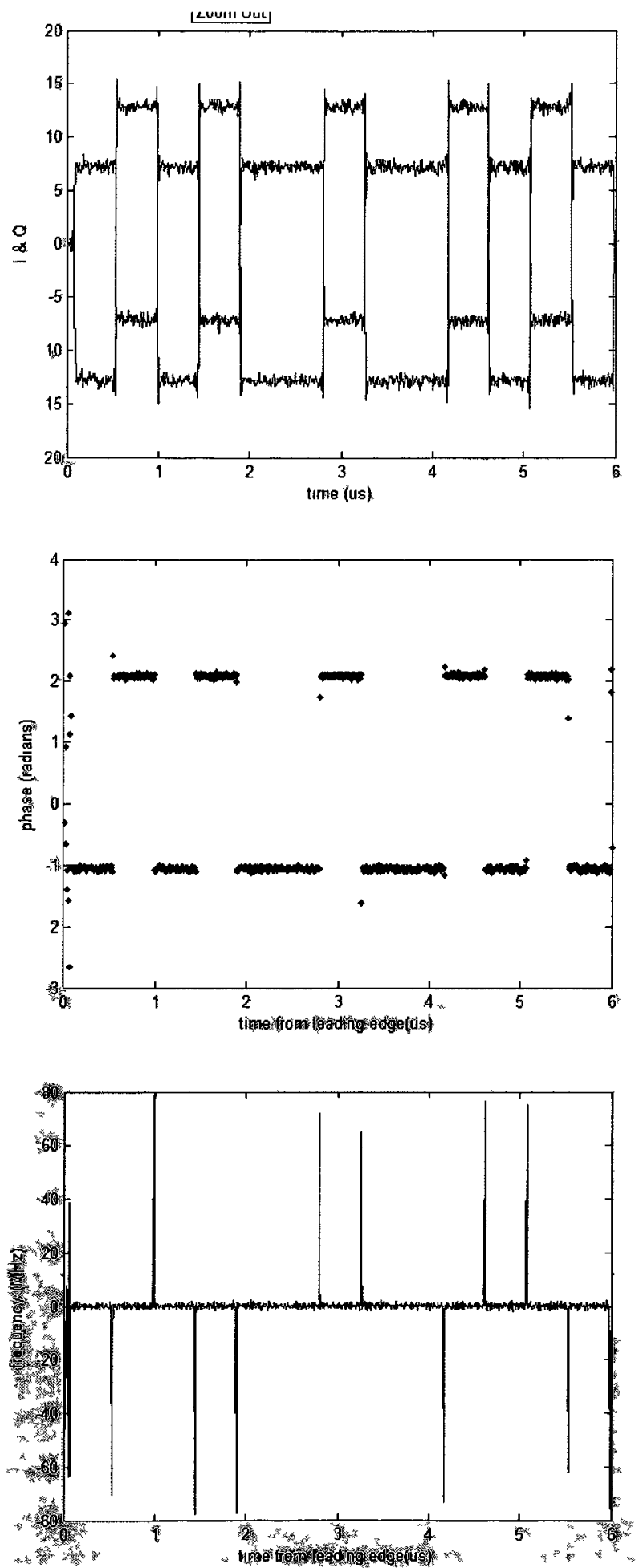

Figure 5.6: Phase-Shift Keying (PSK) $(\mathrm{SNR}=21 \mathrm{~dB})$ 
Reverting back to the simulated scenario in the beginning of this chapter, the five clusters that resulted following the interpulse clustering will now be sorted using the intrapulse information. The second stage of the offline solution will be used for verifying whether each of the five clusters do in fact contain pulses from only one emitter. For four of the five clusters, the Emitter Number Estimation algorithm would provide a resulting emitter count of one, but it is known that the fifth cluster actually contains pulses from four different emitters. To verify the functionality of the Emitter Number Estimation algorithm, it will be tested using a variety of different combinations of pulse modulations. For the testing, each of the four emitters will be assigned distinct IMOP characteristics. The modulations that will be included in the analysis are outlined in Table 5.2, and the randomly selected testing combinations are displayed in Table 5.3. Each test case combination will be simulated with four different signal-to-noise ratios in order to show how the eigenvalues and the Information Theoretic Criteria fluctuate.

\begin{tabular}{|c|c|}
\hline Modulation & Component Modulated \\
\hline LFM 1:0-5 MHz & Frequency \\
\hline LFM 2: $0-10 \mathrm{MHz}$ & Frequency \\
\hline LFM 3: $0-20 \mathrm{MHz}$ & Frequency \\
\hline LFM 4:0 $-30 \mathrm{MHz}$ & Frequency \\
\hline LFM 5: $0-40 \mathrm{MHz}$ & Frequency \\
\hline FSK 1: $-5,10,0,-10,0,10 \mathrm{MHz}$ & Frequency \\
\hline FSK 2: $5,0,-10,-5,0,5 \mathrm{MHz}$ & Frequency \\
\hline PSK 1: +-+-++-++-+-+ & Phase \\
\hline PSK 2: ++-+-+-+-+-++ & Phase \\
\hline NOMOD: No Modulation & None \\
\hline
\end{tabular}

Table 5.2: Modulated Waveforms Used For Testing 


\begin{tabular}{|c|c|}
\hline Test Case & Waveforms \\
\hline A & LFM 1, LFM 2, LFM 3, LFM 4 \\
\hline B & LFM 5, FSK 1, FSK 2, NOMOD \\
\hline C & FSK 1, FSK 2, PSK 1, PSK 2 \\
\hline
\end{tabular}

Table 5.3: Test Case Combinations

Tables 5.4, 5.5, and 5.6 show the results for the various test case and SNR combinations. The eigenvalues obtained from the sample covariance matrix are sorted in descending order and the ten largest eigenvalues are used for the AIC and MDL calculations. When observing the eigenvalues, it is clear that the four largest eigenvalues are significantly larger than the remaining ones in all of the tables. Although this observation can be made visually in many cases, a function is required to quantify the result in situations where there is not as much variation in the eigenvalues. In this thesis, the AIC and MDL functions are used for this purpose. The number of emitters is determined by choosing the value of $\mathrm{k}$ for which the AIC or the MDL is minimized [20]. For Test Cases A, B and C, the value of $\mathrm{k}$ that minimized both the MDL and the AIC was four in all of the simulations except for the one that included Test Case C with an SNR of $21 \mathrm{~dB}$. Since Stage 2 is only used to decide whether a cluster contains more than one emitter, there is not as much concern over the slight overestimate that is obtained. The simulations in this stage were not largely affected by variations in the SNR. A reduction in the SNR lead to an increased magnitude for the six smaller eigenvalues. Following the emitter number estimation, it is evident that the pulses in the cluster originated from more than one emitter. This cluster may now be forwarded to Stage 3, where the Pulse Comparator will deinterleave the pulses using the intrapulse parameters. 


\begin{tabular}{|c|c|c|c|}
\hline $\mathbf{k}$ & Eigenvalues & AIC & MDL \\
\hline 0 & 4669.782 & 97515.007 & 48757.503 \\
\hline 1 & 4028.536 & 95282.439 & 47688.343 \\
\hline 2 & 3786.089 & 92380.946 & 46279.759 \\
\hline 3 & 3570.022 & 86701.01 & 43476.994 \\
\hline 4 & 0.42 & 137.71 & 227.586 \\
\hline 5 & 0.403 & 153.546 & 262.786 \\
\hline 6 & 0.394 & 169.335 & 293.001 \\
\hline 7 & 0.384 & 182.218 & 316.805 \\
\hline 8 & 0.378 & 192.02 & 334.107 \\
\hline 9 & 0.376 & 198 & 344.537 \\
\hline
\end{tabular}

\begin{tabular}{|c|c|c|c|}
\hline $\mathbf{k}$ & Eigenvalues & AIC & MDL \\
\hline 0 & 4671.287 & 74619.411 & 37309.706 \\
\hline 1 & 4024.82 & 72387.672 & 36240.953 \\
\hline 2 & 3777.827 & 69496.504 & 34837.538 \\
\hline 3 & 3575.971 & 63863.819 & 32058.398 \\
\hline 4 & 2.516 & 134.437 & 225.949 \\
\hline 5 & 2.473 & 153.593 & 262.809 \\
\hline 6 & 2.416 & 169.526 & 293.098 \\
\hline 7 & 2.372 & 182.55 & 316.975 \\
\hline 8 & 2.331 & 192.123 & 334.158 \\
\hline 9 & 2.296 & 198 & 344.537 \\
\hline
\end{tabular}

(a) Emitter Number Estimation Test Case A (SNR =(b) Emitter Number Estimation Test Case A (SNR = $21 \mathrm{~dB})$ $13 \mathrm{~dB})$

\begin{tabular}{|c|c|c|c|}
\hline $\mathbf{k}$ & Eigenvalues & AIC & MDL \\
\hline 0 & 4675.918 & 56886.607 & 28443.303 \\
\hline 1 & 4050.06 & 54685.372 & 27389.809 \\
\hline 2 & 3798.334 & 51810.897 & 25994.735 \\
\hline 3 & 3584.106 & 46237.975 & 23245.477 \\
\hline 4 & 10.248 & 133.449 & 225.455 \\
\hline 5 & 10.078 & 153.251 & 262.639 \\
\hline 6 & 9.946 & 169.79 & 293.229 \\
\hline 7 & 9.766 & 182.651 & 317.021 \\
\hline 8 & 9.521 & 192.036 & 334.115 \\
\hline 9 & 9.443 & 198 & 344.537 \\
\hline
\end{tabular}

\begin{tabular}{|c|c|c|c|}
\hline $\mathbf{k}$ & Eigenvalues & AIC & MDL \\
\hline 0 & 4709.731 & 39915.152 & 19957.576 \\
\hline 1 & 4065.067 & 37764.168 & 18929.207 \\
\hline 2 & 3825.715 & 35002.34 & 17590.456 \\
\hline 3 & 3600.475 & 29706.648 & 14979.813 \\
\hline 4 & 40.488 & 135.787 & 226.625 \\
\hline 5 & 40.022 & 154.566 & 263.296 \\
\hline 6 & 38.542 & 169.378 & 293.023 \\
\hline 7 & 38.028 & 182.596 & 316.994 \\
\hline 8 & 37.245 & 192.082 & 334.138 \\
\hline 9 & 36.784 & 198 & 344.537 \\
\hline
\end{tabular}

(c) Emitter Number Estimation Test Case A (SNR =(d) Emitter Number Estimation Test Case A (SNR = $7 \mathrm{~dB})$ 


\begin{tabular}{|c|c|c|c|}
\hline $\mathbf{k}$ & Eigenvalues & AIC & MDL \\
\hline 0 & 6172.494 & 97769.954 & 48884.977 \\
\hline 1 & 4158.807 & 93382.458 & 46738.353 \\
\hline 2 & 3233.552 & 88902.769 & 44540.671 \\
\hline 3 & 2394.754 & 81516.425 & 40884.701 \\
\hline 4 & 0.415 & 133.738 & 225.599 \\
\hline 5 & 0.407 & 153.076 & 262.551 \\
\hline 6 & 0.398 & 169.387 & 293.028 \\
\hline 7 & 0.395 & 182.782 & 317.087 \\
\hline 8 & 0.385 & 192.081 & 334.137 \\
\hline 9 & 0.38 & 198 & 344.537 \\
\hline
\end{tabular}

\begin{tabular}{|c|c|c|c|}
\hline $\mathbf{k}$ & Eigenvalues & AIC & MDL \\
\hline 0 & 6179.121 & 74893.885 & 37446.943 \\
\hline 1 & 4155.27 & 70510.914 & 35302.58 \\
\hline 2 & 3237.409 & 66058.804 & 33118.688 \\
\hline 3 & 2400.111 & 58718.562 & 29485.77 \\
\hline 4 & 2.538 & 134.727 & 226.095 \\
\hline 5 & 2.484 & 154.079 & 263.053 \\
\hline 6 & 2.449 & 170.635 & 293.652 \\
\hline 7 & 2.431 & 183.584 & 317.488 \\
\hline 8 & 2.35 & 192.213 & 334.203 \\
\hline 9 & 2.303 & 198 & 344.537 \\
\hline
\end{tabular}

(a) Emitter Number Estimation Test Case B (SNR =(b) Emitter Number Estimation Test Case B (SNR = $21 \mathrm{~dB})$ $13 \mathrm{~dB})$

\begin{tabular}{|c|c|c|c|}
\hline $\mathbf{k}$ & Eigenvalues & AIC & MDL \\
\hline 0 & 6177.337 & 57622.678 & 28811.339 \\
\hline 1 & 4173.93 & 53276.845 & 26685.546 \\
\hline 2 & 3236.398 & 48839.067 & 24508.82 \\
\hline 3 & 2397.478 & 41625.113 & 20939.045 \\
\hline 4 & 10.067 & 135.35 & 226.406 \\
\hline 5 & 9.841 & 153.819 & 262.922 \\
\hline 6 & 9.571 & 169.641 & 293.155 \\
\hline 7 & 9.454 & 182.905 & 317.148 \\
\hline 8 & 9.324 & 192.383 & 334.288 \\
\hline 9 & 9.076 & 198 & 344.537 \\
\hline
\end{tabular}

\begin{tabular}{|c|c|c|c|}
\hline $\mathbf{k}$ & Eigenvalues & AIC & MDL \\
\hline 0 & 6201.749 & 40237.951 & 20118.975 \\
\hline 1 & 4236.429 & 36040.946 & 18067.596 \\
\hline 2 & 3277.935 & 31745.812 & 15962.192 \\
\hline 3 & 2408.063 & 24975.218 & 12614.098 \\
\hline 4 & 40.642 & 135.305 & 226.384 \\
\hline 5 & 39.848 & 154.469 & 263.248 \\
\hline 6 & 39.213 & 170.657 & 293.663 \\
\hline 7 & 38.611 & 183.364 & 317.378 \\
\hline 8 & 37.685 & 192.373 & 334.283 \\
\hline 9 & 36.696 & 198 & 344.537 \\
\hline
\end{tabular}

(c) Emitter Number Estimation Test Case B (SNR =(d) Emitter Number Estimation Test Case B (SNR = 


\begin{tabular}{|c|c|c|c|}
\hline $\mathbf{k}$ & Eigenvalues & AIC & MDL \\
\hline 0 & 5195.503 & 96410.268 & 48205.134 \\
\hline 1 & 4281.77 & 93401.228 & 46747.737 \\
\hline 2 & 3403.406 & 89438.987 & 44808.78 \\
\hline 3 & 2937.657 & 83163.454 & 41708.216 \\
\hline 4 & 0.588 & 290.044 & 303.753 \\
\hline 5 & 0.453 & 173.277 & 272.651 \\
\hline 6 & 0.404 & 171.043 & 293.856 \\
\hline 7 & 0.391 & 182.761 & 317.076 \\
\hline 8 & 0.383 & 192.133 & 334.163 \\
\hline 9 & 0.377 & 198 & 344.537 \\
\hline
\end{tabular}

\begin{tabular}{|c|c|c|c|}
\hline $\mathbf{k}$ & Eigenvalues & AIC & MDL \\
\hline 0 & 5199.021 & 74437.421 & 37218.711 \\
\hline 1 & 4279.712 & 71431.396 & 35762.822 \\
\hline 2 & 3405.064 & 67485.673 & 33832.123 \\
\hline 3 & 2939.917 & 61240.292 & 30746.635 \\
\hline 4 & 2.603 & 139.899 & 228.681 \\
\hline 5 & 2.539 & 155.867 & 263.946 \\
\hline 6 & 2.43 & 169.432 & 293.051 \\
\hline 7 & 2.372 & 182.318 & 316.855 \\
\hline 8 & 2.333 & 192.032 & 334.113 \\
\hline 9 & 2.316 & 198 & 344.537 \\
\hline
\end{tabular}

(a) Emitter Number Estimation Test Case C (SNR =(b) Emitter Number Estimation Test Case C (SNR = $21 \mathrm{~dB}$ ) $13 \mathrm{~dB}$ )

\begin{tabular}{|c|c|c|c|}
\hline $\mathbf{k}$ & Eigenvalues & AIC & MDL \\
\hline 0 & 5211.628 & 57172.831 & 28586.415 \\
\hline 1 & 4299.165 & 54194.475 & 27144.361 \\
\hline 2 & 3415.143 & 50282.384 & 25230.478 \\
\hline 3 & 2954.867 & 44150.404 & 22201.691 \\
\hline 4 & 10.159 & 136.55 & 227.006 \\
\hline 5 & 9.877 & 154.353 & 263.19 \\
\hline 6 & 9.643 & 170.15 & 293.41 \\
\hline 7 & 9.542 & 183.122 & 317.257 \\
\hline 8 & 9.242 & 192.086 & 334.14 \\
\hline 9 & 9.124 & 198 & 344.537 \\
\hline
\end{tabular}

\begin{tabular}{|c|c|c|c|}
\hline $\mathbf{k}$ & Eigenvalues & AIC & MDL \\
\hline 0 & 5256.744 & 39908.518 & 19954.259 \\
\hline 1 & 4303.196 & 36964.933 & 18529.59 \\
\hline 2 & 3421.766 & 33188.101 & 16683.337 \\
\hline 3 & 2943.534 & 27395.163 & 13824.07 \\
\hline 4 & 40.553 & 135.275 & 226.369 \\
\hline 5 & 39.28 & 153.362 & 262.694 \\
\hline 6 & 38.722 & 169.779 & 293.224 \\
\hline 7 & 37.835 & 182.591 & 316.991 \\
\hline 8 & 37.332 & 192.209 & 334.201 \\
\hline 9 & 36.596 & 198 & 344.537 \\
\hline
\end{tabular}

(c) Emitter Number Estimation Test Case C $(\mathrm{SNR}=(\mathrm{d})$ Emitter Number Estimation Test Case C (SNR = $7 \mathrm{~dB})$ 
The final stage of the offline solution is used to further sort the pulses within the clusters that were identified to contain more than one emitter. It was known that the simulated scenario contained one cluster with pulses from four emitters. This was confirmed by the Emitter Number Estimation algorithm in Stage 2, and the cluster containing pulses from the four tracking radars was forwarded to Stage 3.

The Pulse Comparator is used to perform the sorting in Stage 3 as well as the online solution. Prior to the use of this algorithm, an appropriate threshold for pulse similarity must be selected. For testing purposes, the three test case combinations in Table 5.3 that were used in Stage 2 will be maintained for the current stage. For each test case combination the SNR is also varied. Tables 5.7, 5.8, and 5.9 illustrate the result of the Pulse Comparator analysis. It can be seen that there is a significant difference between pulses of the same modulation and pulses that have differing modulations. This difference becomes less evident as the pulse SNR decreases. Observing the values obtained for the lowest SNR, it can be seen that the pulses with the same intrapulse waveforms obtain a Pulse Comparator result of approximately ten times less than pulses that have varying modulations. Based on these results, a threshold of half the energy of the pulses will be used as the decision-criteria for pulse similarity. This criteria should produce the optimum clustering result for the simulated scenario. Once the threshold is set, the remaining parts of the algorithm will be completed as described in the previous chapter. 


\begin{tabular}{|l|c|c|c|c|}
\hline & LFM 1 & LFM 2 & LFM 3 & LFM 4 \\
\hline LFM 1 & 275.907 & 191290 & 200020 & 202110 \\
\hline LFM 2 & 191290 & 269.332 & 199510 & 203660 \\
\hline LFM 3 & 200020 & 199510 & 286.246 & 201930 \\
\hline LFM 4 & 202110 & 203660 & 201930 & 264.662 \\
\hline
\end{tabular}

(a) Pulse Comparator Test Case A (SNR $=21 \mathrm{~dB})$

\begin{tabular}{|l|c|c|c|c|}
\hline & LFM 1 & LFM 2 & LFM 3 & LFM 4 \\
\hline LFM 1 & 1648.5 & 191700 & 202560 & 204010 \\
\hline LFM 2 & 191700 & 1664.1 & 201210 & 204490 \\
\hline LFM 3 & 202560 & 201210 & 1648.9 & 202850 \\
\hline LFM 4 & 204010 & 204490 & 202850 & 1665.4 \\
\hline
\end{tabular}

(b) Pulse Comparator Test Case A (SNR $=13 \mathrm{~dB})$

\begin{tabular}{|l|c|c|c|c|}
\hline & LFM 1 & LFM 2 & LFM 3 & LFM 4 \\
\hline LFM 1 & 6198.6 & 196690 & 206560 & 206330 \\
\hline LFM 2 & 196690 & 6412.4 & 206350 & 207520 \\
\hline LFM 3 & 206560 & 206350 & 6365.4 & 206780 \\
\hline LFM 4 & 206330 & 207520 & 206780 & 6790.1 \\
\hline
\end{tabular}

(c) Pulse Comparator Test Case A (SNR $=7 \mathrm{~dB})$

\begin{tabular}{|l|c|c|c|c|}
\hline & LFM 1 & LFM 2 & LFM 3 & LFM 4 \\
\hline LFM 1 & 24282 & 216200 & 223410 & 228150 \\
\hline LFM 2 & 216200 & 26799 & 225160 & 227890 \\
\hline LFM 3 & 223410 & 225160 & 24858 & 227930 \\
\hline LFM 4 & 228150 & 227890 & 227930 & 26409 \\
\hline
\end{tabular}

(d) Pulse Comparator Test Case A (SNR $=1 \mathrm{~dB})$

Table 5.7: Pulse Comparator Test Case A 


\begin{tabular}{|l|c|c|c|c|}
\hline & LFM 5 & FSK 1 & FSK 2 & NOMOD \\
\hline LFM 5 & 253.451 & 202570 & 198600 & 203220 \\
\hline FSK 1 & 202570 & 264.62 & 176170 & 140480 \\
\hline FSK 2 & 198600 & 176170 & 272.5489 & 139370 \\
\hline NOMOD & 203220 & 140480 & 139370 & 257.668 \\
\hline
\end{tabular}

(a) Pulse Comparator Test Case B (SNR $=21 \mathrm{~dB})$

\begin{tabular}{|l|c|c|c|c|}
\hline & LFM 5 & FSK 1 & FSK 2 & NOMOD \\
\hline LFM 5 & 1656 & 203340 & 199970 & 205150 \\
\hline FSK 1 & 203340 & 1670.7 & 177390 & 142050 \\
\hline FSK 2 & 199970 & 177390 & 1525.6 & 140980 \\
\hline NOMOD & 205150 & 142050 & 140980 & 1585.5 \\
\hline
\end{tabular}

(b) Pulse Comparator Test Case B (SNR $=13 \mathrm{~dB})$

\begin{tabular}{|l|c|c|c|c|}
\hline & LFM 5 & FSK 1 & FSK 2 & NOMOD \\
\hline LFM 5 & 6350 & 207520 & 203180 & 209210 \\
\hline FSK 1 & 207520 & 6600.1 & 179740 & 145930 \\
\hline FSK 2 & 203180 & 179740 & 6398.8 & 144780 \\
\hline NOMOD & 209210 & 145930 & 144780 & 6915.9 \\
\hline
\end{tabular}

(c) Pulse Comparator Test Case B (SNR $=7 \mathrm{~dB})$

\begin{tabular}{|l|c|c|c|c|}
\hline & LFM 5 & FSK 1 & FSK 2 & NOMOD \\
\hline LFM 5 & 25628 & 223810 & 224700 & 225940 \\
\hline FSK 1 & 223810 & 25841 & 207180 & 161560 \\
\hline FSK 2 & 224700 & 207180 & 28437 & 171580 \\
\hline NOMOD & 225940 & 161560 & 171580 & 25371 \\
\hline
\end{tabular}

(d) Pulse Comparator Test Case B (SNR $=1 \mathrm{~dB})$

Table 5.8: Pulse Comparator Test Case B 


\begin{tabular}{|l|l|l|l|l|}
\hline & FSK 1 & FSK 2 & PSK 1 & PSK 2 \\
\hline FSK 1 & 257.274 & 176660 & 188650 & 202470 \\
\hline FSK 2 & 176660 & 263.519 & 198600 & 196740 \\
\hline PSK 1 & 188650 & 198600 & 307.335 & 158860 \\
\hline PSK 2 & 202470 & 196740 & 158860 & 260.019 \\
\hline
\end{tabular}

(a) Pulse Comparator Test Case C (SNR $=21 \mathrm{~dB})$

\begin{tabular}{|l|c|c|c|c|}
\hline & FSK 1 & FSK 2 & PSK 1 & PSK 2 \\
\hline FSK 1 & 1752.8 & 178530 & 189780 & 204420 \\
\hline FSK 2 & 178530 & 1710.9 & 198570 & 197100 \\
\hline PSK 1 & 189780 & 198570 & 1533 & 159820 \\
\hline PSK 2 & 204420 & 197100 & 159820 & 1586.8 \\
\hline
\end{tabular}

(b) Pulse Comparator Test Case C (SNR $=13 \mathrm{~dB})$

\begin{tabular}{|l|l|l|l|l|}
\hline & FSK 1 & FSK 2 & PSK 1 & PSK 2 \\
\hline FSK 1 & 6484.7 & 183990 & 194410 & 208060 \\
\hline FSK 2 & 183990 & 6716.4 & 204920 & 201860 \\
\hline PSK 1 & 194410 & 204920 & 6602.6 & 166910 \\
\hline PSK 2 & 208060 & 201860 & 166910 & 6299.5 \\
\hline
\end{tabular}

(c) Pulse Comparator Test Case C (SNR $=7 \mathrm{~dB})$

\begin{tabular}{|l|c|c|c|c|}
\hline & FSK 1 & FSK 2 & PSK 1 & PSK 2 \\
\hline FSK 1 & 26551 & 205460 & 212840 & 220700 \\
\hline FSK 2 & 205460 & 26764 & 218650 & 217910 \\
\hline PSK 1 & 212840 & 218650 & 25433 & 179880 \\
\hline PSK 2 & 220700 & 217910 & 179880 & 25542 \\
\hline
\end{tabular}

(d) Pulse Comparator Test Case C $(\mathrm{SNR}=1 \mathrm{~dB})$

Table 5.9: Pulse Comparator Test Case C 


\section{Chapter Summary}

This chapter began with the introduction of a simulated scenario that contained a coordinate system centered by the ELINT or ES receiver, and surrounded by a number of different radar systems. To test the first stage, each radar was assigned specific interpulse parameters, which were sorted using the K-Means technique. Then, a number of test cases were defined to test the Emitter Number Estimation algorithm and the Pulse Comparator algorithm. Each test case consisted of different intrapulse modulations and varying signal-to-noise ratios. Following the completion of the testing, it was shown that both the offline solution and online solution would be effective at deinterleaving the simulated pulses in this thesis. 


\section{Chapter 6}

\section{Conclusion}

The fields of Electronic Warfare and Signal Intelligence are continuously evolving in conjunction with the evolution of radar systems. The complexity of modern radar transmission techniques and pulse waveforms makes it very difficult for the conventional interpulse deinterleavers to cluster radar pulses effectively. There is a large probability that current high density electromagnetic environments will contain emitters with similar pulse characteristics. As a result, the conventional deinterleavers must be supplemented with algorithms that can account for their shortfalls. This combination of algorithms provides a solution that is more flexible, and it is capable of producing an accurate picture of the environment surrounding the EW receiver.

This thesis proposed two possible solutions that may be used to solve some of the current deinterleaving problems. Both solutions use the IMOP intrapulse characteristics to provide improved clustering performance. The first solution is offline, and it could be used in an ELINT application to sort a large number of 
pulses. The three algorithms used as part of the offline solution were each obtained from other research papers and combined to form the ELINT pulse deinterleaving method in this thesis. The second proposed solution is online, and it could be used in an ES application where pulses must be sorted in real-time as they are received. The operation of both solutions was illustrated using a simulated scenario which contained an EW receiver surrounded by a number of different radar systems. After each radar system was assigned both interpulse and intrapulse parameters, various test cases were created to verify the operation of the algorithms used in the solutions. Following the testing and simulations, each solution was able to deinterleave the pulses into individual groups that belonged to each emitter.

Radar pulse deinterleaving has been and will continue to be a heavily researched topic. The offline solution that is outlined in this thesis illustrates a general deinterleaving technique that has been adopted in many research papers. A multistage procedure is used with a combination of both the interpulse and intrapulse parameters to sort the received radar pulses.

The first stage consists of an algorithm that sorts the pulses based on a subset of selected interpulse parameters. As mentioned earlier in this thesis, there are a number of different methods that may be used to complete this task, and in some cases, certain algorithms may be more effective than others. Future research may be used to examine the various existing interpulse algorithms, and to allocate them to specific applications where they may be most effective. The third stage of the offline solution compares the similarity between pulses using the intrapulse characteristics. One of the drawbacks of this Pulse Comparator algorithm is that it 
requires a threshold to be chosen by the user. Research may be conducted to obtain a way to refine this threshold so that it is updated adaptively.

The solutions in this thesis only use the IMOP intrapulse characteristics to perform the pulse deinterleaving. It is also possible to use the UMOP intrapulse characteristics to differentiate between pulses from multiple emitters. In order to validate the expected performance of the solutions discussed in this thesis as well as any future research, it would be extremely valuable to conduct the testing using measured field data. 


\section{Bibliography}

[1] D. Adamy, EW 102. Boston, MA: Artech House, 2004.

[2] R. Wiley, ELINT: The Interception and Analysis of Radar Signals. Norwood, MA: Artech House, 2006.

[3] N. Polmar, "The u.s. navy electronics warfare (part 1)," United States Naval Institute Proceedings, 1979.

[4] L. Langley, "Specific emitter identification (sei) and classical parameter fusion technology," IEEE WESCON/93 Conference Record, pp. 377 - 381, 1993.

[5] A. Kawalec and R. Owczarek, "Specific emitter identification using intrapulse data," European Radar Conference, pp. 249 - 252, 2004.

[6] M. I. Skolnik, Introduction to Radar Systems. Boston, MA: McGraw-Hill, 2001.

[7] D. Adamy, Introduction to Electronic Warfare Modeling and Simulation. Norwood, MA: Artech House, 2003.

[8] J. Liu, S. Gao, Z.-Q. Luo, T. Davidson, and J. P. Lee, "The minimum description length criterion applied to emitter number detection and pulse classification," IEEE Workshop Statistical Signal and Array Processing, pp. 172 $-175,1998$. 
[9] J. Liu, J. P. Lee, L. Li, Z.-Q. Luo, and M. Wong, "Online clustering algorithms for radar emitter classification," IEEE Transactions on Pattern Analysis and Machine Intelligence, vol. 27, no. 8, pp. 1185 - 1196, 2005.

[10] M. Wong, Z.-Q. Luo, J. Liu, J. P. Lee, and S. Gao, "Radar emitter classification using intrapulse data," International Journal of Electronics and Communications, vol. 53, no. 6, pp. $324-332,1999$.

[11] S. Howard, "Estimation and correlation of radar pulse modulations for electronic support measures," IEEE Aerospace Conference, vol. 5, 2003.

[12] Y. Zhou and J. P. Lee, "Combining clustering techniques and information theoretic criteria based approach for emitter number detection in esm applications," Signals, Systems and Computers Conference, pp. 474-478, 1999.

[13] J. Lunden and V. Koivunen, "Robust estimation of radar pulse modulation," IEEE International Symposium on Signal Processing and Information Technology, pp. $271-276,2006$.

[14] N. A. W. Center, Electronic Warfare and Radar Systems Engineering Handbook. Point Mugu, CA: Electronic Warfare Division, 1999.

[15] R. Duda, P. Hart, and D. Stork, Pattern Classification. New York: John Wiley and Sons, 2001.

[16] Q. Guo, W. Chen, X. Zhang, Z. Li, and D. Guan, "Signal sorting based on svc \& k-means clustering in esm systems," ICONIP (2), pp. 596-603, 2006.

[17] P. Hansson, "Analysis of some methods for deinterleaving of pulse trains," Master's thesis, KTH Electrical Engineering, 2007.

[18] G. Zhang, H. Rong, and W. Jin, "Intra-pulse modulation recognition of unknown radar emitter signals using support vector clustering," in Fuzzy Systems and Knowledge Discovery, vol. 4223, no. 48, pp. 420 - 429, 2006. 
[19] E. Granger, Y. Savaria, P. Lavoie, and M.-A. Cantin, "A comparison of self-organizing neural networks for fast clustering of radar pulses," Signal Processing, vol. 64, pp. 249 - 269, 1998.

[20] M. Wax and T. Kailath, "Detection of signals by information theoretic criteria," IEEE Transactions on Acoustics, Speech, and Signal Processing, vol. 33, no. 2, pp. $387-392,1985$.

[21] H.-J. Du, 2009. Personal Communication.

[22] H. Akaike, "A new look at the statistical model identification," IEEE Transactions $A C$, vol. 6, pp. $716-723,1974$.

[23] J. Rissanen, "Modeling by the shortest description," Automatica, vol. 14, pp. $465-471,1978$.

[24] G. Schwartz, "Estimating the dimension of a model," Ann. Stat, vol. 6, pp. 461 $-464,1978$.

[25] Y. Zhou, "Emitter number estimation from pulse trains using the gic," IEEE Transactions on Aerospace and Electronic Systems, vol. 41, no. 3, pp. 809 $818,2005$.

[26] Y. Yin and P. Krishnaiah, "On some nonparametric methods for detection of the number of signals," IEEE Transactions on Acoustics, Speech, and Signal Processing, vol. 35, no. 11, pp. 1533 - 1538, 1987.

[27] H. Lee and F. Li, "A novel approach for detecting the number of emitters in a cluster," IEEE Transactions on Acoustics, Speech, and Signal Processing, vol. 1 , pp. $261-264,1993$.

[28] Y. Zhou and J. P. Lee, "A minimum description length emitter number detection approach for intra-pulse analysis," in Proceedings of the IEEE Pacific 
Rim Conference on Communications, Computers and Signal Processing, pp. $548-551,1999$.

[29] T. W. Anderson, "Asymptotic theory for principal component analysis," Ann. J. Stat., vol. 34, pp. $122-148,1963$. 
Appendices 


\section{Appendix A}

\section{The Radar Equation}

The radar equation is one of the primary methods of assessing the expected performance of a radar system during the design phase [6]. The derivation for the radar equation begins by starting with an isotropic radiator which radiates energy equally in all directions. The formula for the nondirectional power density, $S_{n}$, is:

$$
S_{n}=\frac{P_{t}}{4 \pi R_{1}^{2}}
$$

where:

- $P_{t}=$ transmitted peak power

- $R_{1}=$ range to the target

If the radar energy is concentrated in one direction then there is an increase in the power density in that particular direction as well as a decrease in the power density for all other directions. The directional power density, $S_{d}$, is:

$$
S_{d}=S_{n} G=\frac{P_{t} G}{4 \pi R_{1}^{2}}
$$

where: 
- $G=$ gain of the antenna

The amount of power that is reflected from a target depends on the target's radar cross-section. The reflected power, $P_{e}$, is defined as:

$$
P_{e}=S_{d} \sigma=\frac{P_{t} G \sigma}{4 \pi R_{1}^{2}}
$$

where:

- $\sigma=$ radar cross-section of the target

Once the energy hits the target, it acts as an isotropic radiator, radiating the energy in all directions. As a result, the power density that exists at the radar receiver, $S_{r}$, is:

$$
S_{r}=\frac{P_{e}}{4 \pi R_{2}^{2}}=\frac{P_{t} G \sigma}{\left(4 \pi R_{1}^{2}\right)\left(4 \pi R_{2}^{2}\right)}
$$

where:

- $R_{2}=$ range of antenna from target

The amount of power that is received by the antenna depends on the effective area of the antenna. The received power, $P_{r}$, is:

$$
P_{r}=S_{r} A_{e}=\frac{P_{t} G \sigma A_{e}}{\left(4 \pi R_{1}^{2}\right)\left(4 \pi R_{2}^{2}\right)}
$$

where:

- $A_{e}=$ effective antenna area

To continue the derivation the following formula will be used:

$$
A_{e}=\frac{G \lambda^{2}}{4 \pi}
$$

where: 
- $\lambda=$ transmitted wavelength

Substituting in for $A_{e}$ and making the assumption that $R_{1}=R_{2}$, since the transmitter and receiver are co-located, the received power, $P_{r}$, becomes:

$$
P_{r}=\frac{P_{t} G^{2} \lambda^{2} \sigma}{(4 \pi)^{3} R^{4}}
$$

Although this Appendix will not provide anymore detail on the radar equation derivation, it is worth noting that there are other parameters that may contribute to the radar equation which can be used to obtain a more realistic estimate of performance. Some of these parameters relate to internal and external losses, influence of the Earth's surface and ground reflections.

One important detail when relating the radar equation to Electronic Warfare applications is that the energy received by the EW receiver is dependent only on $R^{2}$ rather than $R^{4}$. This is because the energy only travels in one direction before it is received. This provides an advantage to the EW receiver, since it does not have to be as sensitive as the radar receiver. The power received by the EW receiver may be calculated using the one-way radar equation as follows:

$$
P_{r}=\frac{P_{t} G_{t} G_{r} \lambda^{2}}{(4 \pi R)^{2}}
$$

where:

- $P_{t}=$ transmitted peak power

- $G_{t}=$ gain of the transmitting antenna

- $G_{r}=$ gain of the EW receiver

- $\lambda=$ transmitted wavelength

- $R=$ range from the radar transmitter to the EW receiver 


\section{Appendix B}

\section{Signal-to-Noise Variations in LFM Pulse}



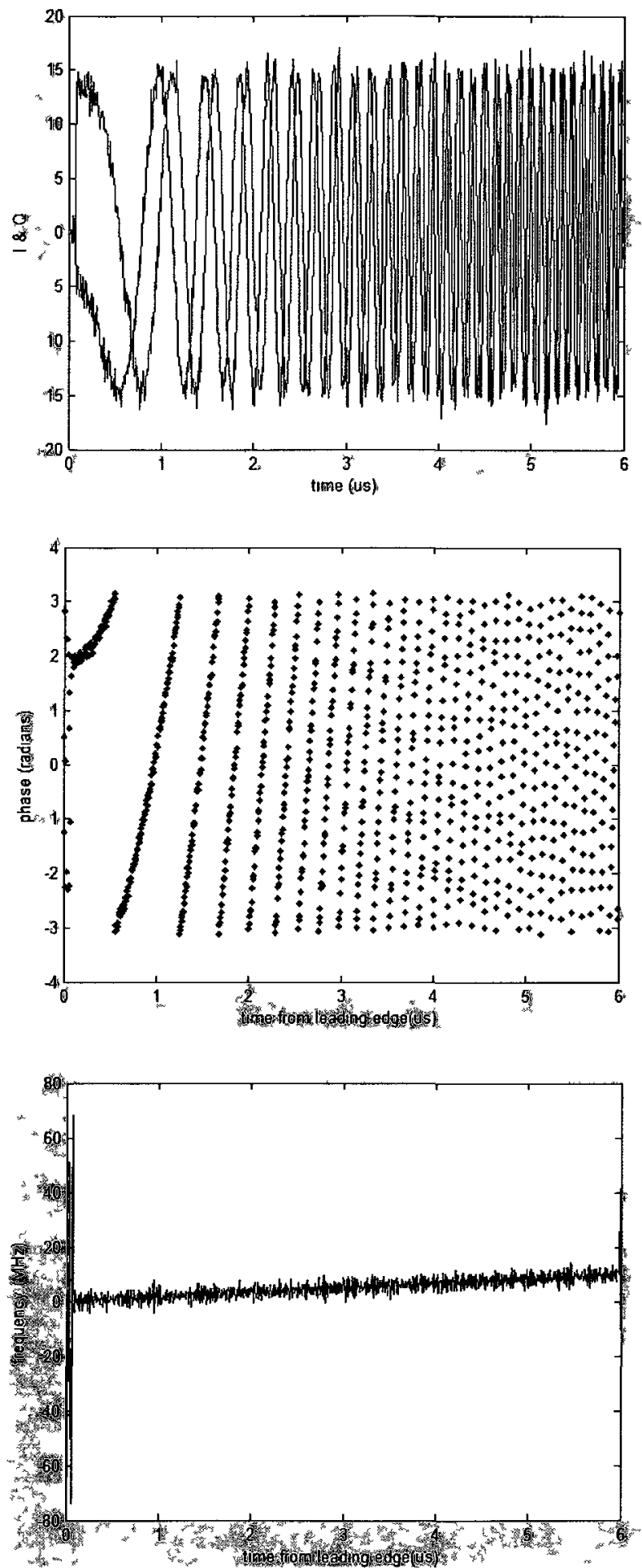

Figure B.1: Linear Frequency Modulation $(\mathrm{LFM})(\mathrm{SNR}=13 \mathrm{~dB})$ 

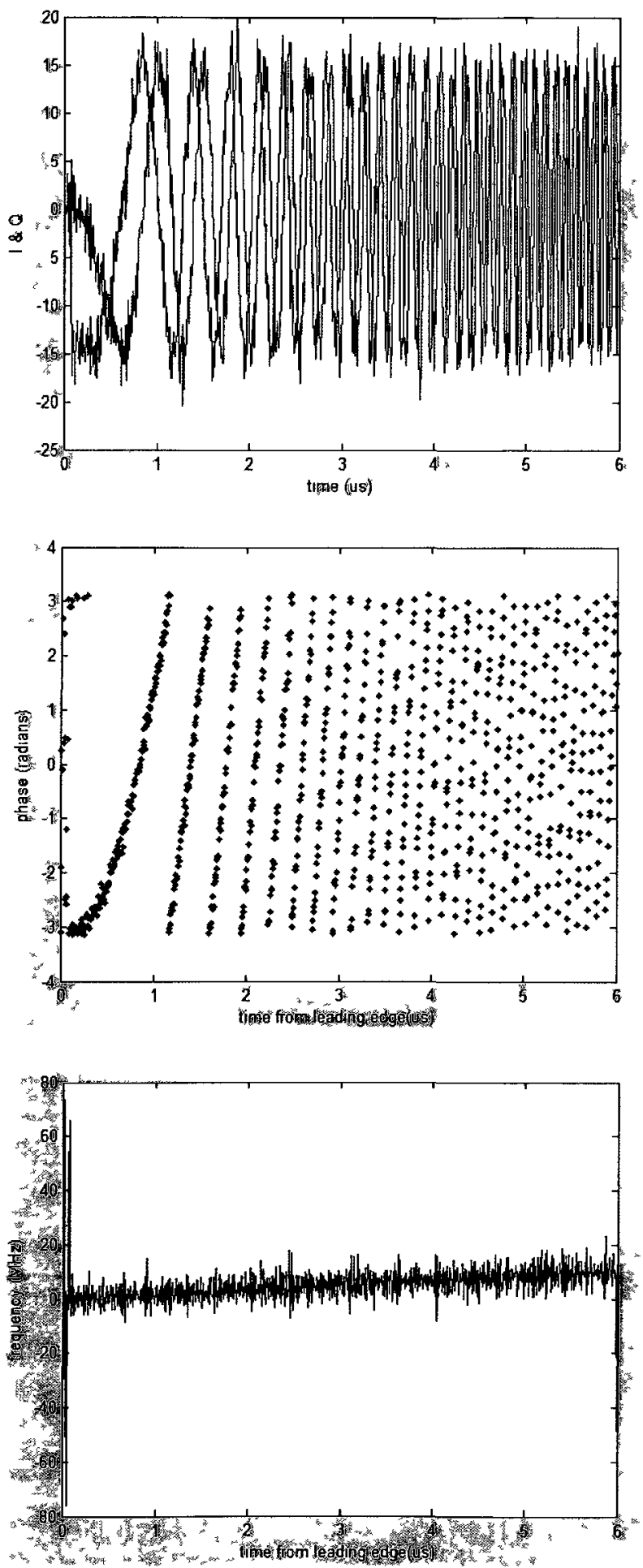

Figure B.2: Linear Frequency Modulation (LFM) $(\mathrm{SNR}=7 \mathrm{~dB})$ 

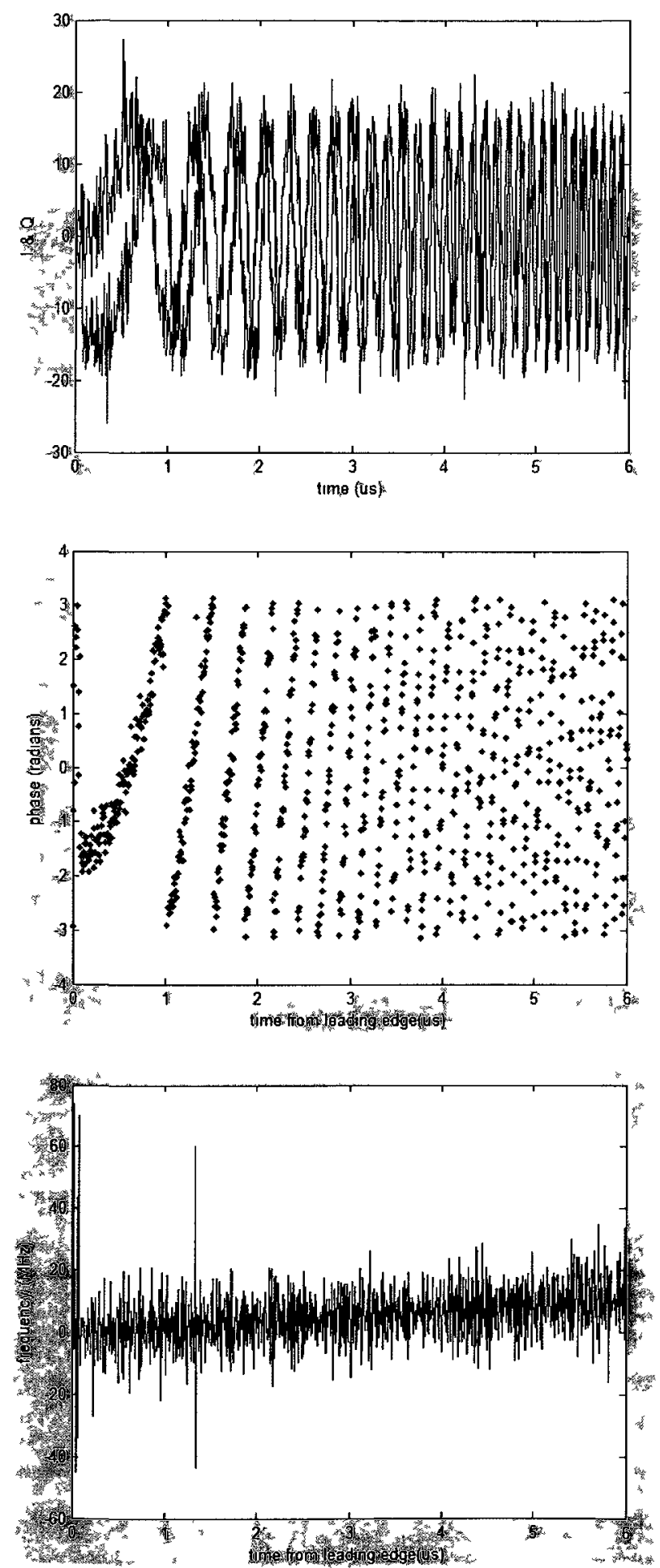

Figure B.3: Linear Frequency Modulation $(\mathrm{LFM})(\mathrm{SNR}=1 \mathrm{~dB})$ 